\title{
Steady states of active Brownian particles interacting with boundaries
}

\author{
Caleb G. Wagner ${ }^{1}$, Michael F. Hagan², Aparna Baskaran² \\ ${ }^{1}$ Mechanical and Materials Engineering, University of Nebraska-Lincoln, Lincoln, NE 68588 \\ ${ }^{2}$ Martin Fisher School of Physics, Brandeis University, Waltham, Massachusetts 02453, USA
}

\begin{abstract}
An active Brownian particle is a minimal model for a self-propelled colloid in a dissipative environment. Experiments and simulations show that, in the presence of boundaries and obstacles, active Brownian particle systems approach nontrivial nonequilibrium steady states with intriguing phenomenology, such as accumulation at boundaries, ratchet effects, and long-range depletion interactions. Nevertheless, theoretical analysis of these phenomena has proven difficult. Here we address this theoretical challenge in the context of non-interacting particles in two dimensions, basing our analysis on the steady-state Smoluchowski equation for the 1-particle distribution function. Our primary result is an approximation strategy that explicitly connects asymptotic solutions of the Smoluchowski equation to boundary conditions. We test this approximation against the exact analytic solution in a $2 \mathrm{~d}$ planar geometry as well as numerical solutions in circular and elliptic geometries. We find good agreement so long as the boundary conditions do not vary too rapidly with respect to the persistence length of particle trajectories. Our results are relevant for characterizing long-range flows and depletion interactions in such systems. In particular, our framework shows how such behaviors are connected to the breaking of detailed balance at the boundaries.
\end{abstract}

\section{Introduction}

Active matter describes a class of systems that are maintained far from equilibrium by driving forces acting on the constituent particles [50, 42, 7, 84, 83, 51, 46, 56, 27, 60]. Experimental realizations span many scales, from the microscopic and colloidal to the macroscopic. Examples on the microscopic level are reconstituted biopolymers and molecular motors [57], bacterial suspensions [21, 37], and synthetic self-propelled colloids [20, 8, 49]. On larger scales, many macrobiological systems can be thought of as active matter, including swarming midges [70,12], aggregating fire ants [69], schooling fish $[6,9]$, and flocking birds [2]. There are also examples of macroscopic active systems constructed artificially, such as vibrated self-propelled granular particles [44, 45, 76, 34, 77, 59] and small robots [58, $31,19,17]$. Naturally, biological applications are a significant motivation for studying active matter. At an abstract level, active matter is also a promising testing bed for the development of new ideas about nonequilibrium steady states in general.

Active matter steady states exhibit many unusual properties. Examples are the nonexistence of state variables like pressure and temperature [64, 34], violations of extensivity [74], aggregation at boundaries [79, 72, 23, 38, 24], and athermal phase separation [25, 53, 66, 65, 81, 11, 67, 48, 54]. Moreover, while equilibrium systems obey detailed balance and cannot exhibit net currents, this is no longer true out of equilibrium. Strikingly, in active matter systems, currents can occur even without external driving, instead resulting from rectification of the particle-level active driving by way of spatial asymmetries in the boundary conditions $[78,55]$.

At the concrete level, progress toward a fundamental understanding of these unusual phenomena requires minimal models that reproduce characteristically nonequilibrium phenomenology while still being amenable to theoretical treatment. In this work, we focus on a simple, well-studied model for active matter, a non-interacting collection of active Brownian particles (ABPs). This minimal model exhibit several of the features of nonequilibrium steady states mentioned above, including 
accumulation at boundaries, nonlocal probability measures, and long-ranged currents in steady-state. Within the context of this model, we focus on the long-ranged currents observed in active matter steady states. Their existence was first shown by Baek, et al [3], who derived a multipole expansion for the density and current exterior to a fixed, passive inclusion. These solutions do not require external forcing, in agreement with previous simulation results on active ratchet systems [55]. Instead, geometric constraints imposed by boundaries rectify the active driving, inducing currents. As an example, the current exterior to a fixed, elliptical inclusion asymptotically decays as $r^{-2}$, and the density as $r^{-1}$.

While Ref. 3 has shown that long-range, current-carrying solutions do exist, it is not obvious how to connect these solutions with boundary conditions. In this work, we develop an approximation scheme that connects the asymptotic (long-range) behavior of the steady state with boundary conditions. We demonstrate the utility of this scheme by considering planar, circular, and elliptic geometries. In particular, we identify the mechanism by which certain surface-particle interactions generate long-range dipolar and quadrupolar flow fields. We verify our analytical results using two numerical methods: the first, a trajectory-sampling technique based on the particle dynamics and the second, the finite element software PDE2D ${ }^{1}$.

Our framework proves that long-range effects, and in particular long-range currents, are tied with the breaking of detailed balance at the boundaries. We argue that this connection has far-reaching implications for nonequilibrium steady states in general, not just those in active matter. Further, our work provides insight into pathways to construct finite current steady states via the design of boundary conditions. As we provide intuitive and computationally cheap methods to connect boundary conditions to material properties in bulk, they can be used to significantly constrain the space of design possibilities to obtain such steady states. One can then harness these types of long-range density variations and spontaneous flows to engineer active baths with tunable functionality, such as "depletion-induced" longranged forces between passive inclusions that can be either attractive or repulsive depending on the configuration and design [1, 52, 30, 47, 39, 82, 3, 40].

\section{Model definitions}

We consider active Brownian particles in $2 \mathrm{~d}$. The dynamics of the center of mass $\mathbf{r}(t)=$ $(x(t), y(t))$ and $\hat{u}=(\cos \theta, \sin \theta)$, the direction of the self-propulsion velocity, is given by

$$
\begin{aligned}
& \dot{\mathbf{r}}=-\xi^{-1} \nabla V(\mathbf{r})+v_{0} \hat{u}+\sqrt{2 D_{t}} \boldsymbol{\eta}^{\mathrm{T}} \\
& \dot{\theta}=\sqrt{2 D_{r}} \eta^{\mathrm{R}}
\end{aligned}
$$

Here $\xi$ is the friction (units of [mass]/[time]), $V(\mathbf{r})$ is an external potential, $v_{0}$ is the magnitude of the self-propulsion velocity, and $D_{t}$ and $D_{r}$ are the translational and rotational diffusion coefficients. The $\eta$ variables are Gaussian white noise with $\left\langle\eta_{i}(t)\right\rangle=0$ and $\left\langle\eta_{i}(t) \eta_{j}\left(t^{\prime}\right)\right\rangle=\delta_{i j} \delta\left(t-t^{\prime}\right)$. It is also useful to formulate the steady-state statistics of Eqs. (1)-(2) in terms of the associated Fokker-Planck equation for the probability density function $f(\mathbf{r}, \theta)$ :

$$
\ell \hat{u} \cdot \nabla f=-\left(\xi D_{r}\right)^{-1} \nabla \cdot[\nabla V(\mathbf{r}) f]+\left(D_{t} / D_{r}\right) \nabla^{2} f+\partial_{\theta}^{2} f
$$

\footnotetext{
${ }^{1}$ www.pde2d.com
} 


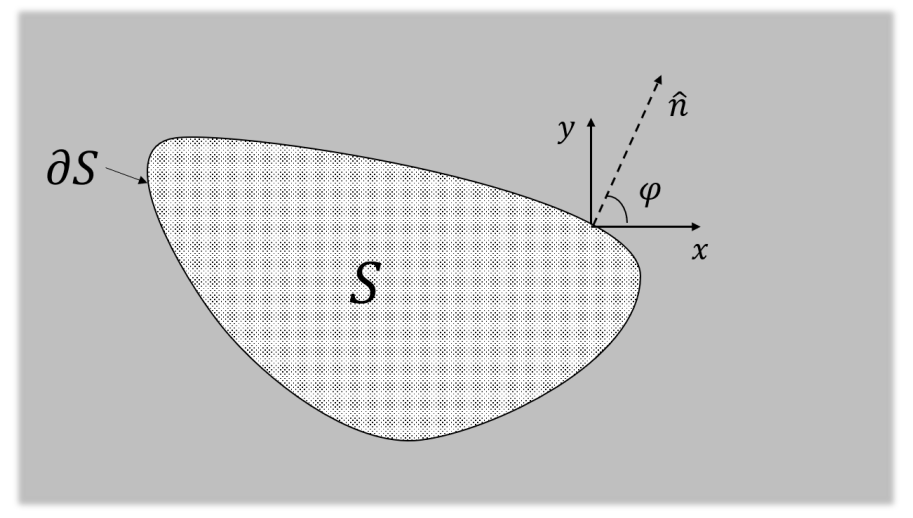

Figure 1: Illustration of the problems of interest in this work. The region $S$ models a fixed inclusion with hard walls. The boundary $\partial S$ is an idealized approximation of the narrow region $\mathbb{B}$ on which $\nabla V(\mathbf{r})$ is steep and repulsive. Outside of $S, \nabla V(\mathbf{r})$ is zero and the ABPs move freely with a uniform density boundary condition at infinity.

where $\ell \equiv v_{0} / D_{r}$ is a length associated with the persistence of particle trajectories in space. Previous work has used this equation as a starting point for various approximate descriptions of ABP steady states. Nevertheless, solutions which are both exact and nontrivial are essentially nonexistent. In this work we first approximate the Fokker-Planck equation itself, isolating the effects of activity by neglecting thermal noise (setting $\left.D_{t}=0\right)$. We also drop the external forcing term by letting $\nabla V(\mathbf{r})$ simulate a hard wall boundary condition. Specifically, we make $\nabla V(\mathbf{r})$ large on a negligible subdomain $\mathbb{B}$, which defines the walls, and 0 otherwise. Then, (3) simplifies to

$$
\ell \hat{u} \cdot \nabla f=\partial_{\theta}^{2} f, \quad \mathbf{r} \notin \mathbb{B}
$$

\section{Method of solution}

Our primary result is a method for solution of (4) based on a decomposition into boundary layer and asymptotic parts. The existence of a boundary layer is motivated by the first-order spatial gradients in (4), which suggest solutions that decay exponentially over the length scale $\ell$. It is useful to schematically write these boundary layer solutions as $\sim e^{-\frac{s}{\epsilon}}$, where $s$ is an appropriate scalar length given in units of the system size, and $\epsilon \equiv \ell / L$ is a dimensionless parameter usually assumed to be small. In this form, we see that the boundary layer solutions are nonanalytic in $\epsilon$. This raises the question of whether solutions exist which are analytic in $\epsilon$, i.e. expressible asymptotically as a series in $\epsilon$ :

$$
f \sim f_{0}+\epsilon f_{1}+\epsilon^{2} f_{2}+\cdots
$$

Such solutions are known to exist for a broad class of integro-differential equations, called "transport equations", that are similar to (4) in abstract structure and model various transport phenomena. In these contexts, asymptotic solutions like (5) carry special physical relevance. In general, the length $\ell$ is an effective mean free path describing the typical distance a particle traverses between scattering events. In contrast to the boundary layer solutions, the asymptotic solutions (5) persist into the bulk and therefore function as a useful basis for coarse-grained, macroscopic descriptions of microscopic 
transport. For instance, in the Boltzmann equation for dilute gases, they generate classical hydrodynamics and its extensions, e.g. the Euler, Navier-Stokes, and Burnett equations [16, 29].

The challenge in extracting the asymptotic solution is identifying proper boundary conditions. In general, this is not possible without solving for the boundary layer as well, since both pieces make a necessary and nontrivial contribution to the boundary condition on the full equation. The usual strategy is to propose approximate or phenomenological boundary conditions: an example from kinetic theory is the "no-slip" boundary condition on the Navier-Stokes equations [18]. In our case there is the added challenge that the approximations used to derive (4) from the physical dynamics constitute a singular limit. For this reason, identifying the boundary conditions on the full distribution function $f(\mathbf{r}, \theta, t)$ is delicate; for a detailed treatment, see Ref. 75.

Our strategy here, by contrast, is to extract the asymptotic solution to (4) without having to solve for the boundary layer. In this section, we sketch this strategy in general terms; sections 4-6 then carry out the analysis in specific cases. Similar analyses for other transport equations can be found in the literature; see, for instance, the linearized Boltzmann equation $[13,14,15,32,36]$, neutron transport[10, 85], and Fokker-Planck equation [43, 80, 35].

Let us begin by considering the steady state solution in the bulk. With $x$ and $y$ in units of $L$, Eq. (4) can be written as

$$
\epsilon\left(\cos \theta \frac{\partial f}{\partial x}+\sin \theta \frac{\partial f}{\partial y}\right)=\frac{\partial^{2} f}{\partial \theta^{2}}
$$

The bulk solution $f_{d}$, which we call the diffusion solution, being an analytic function of $\epsilon$, is assumed to have the asymptotic expansion $f_{d}=f_{0}+\epsilon f_{1}+\epsilon^{2} f_{2}+\cdots$. If $\epsilon=0$, the particles do not self-propel, and their distribution should be uniform. Therefore, we take $f_{0}=c_{0}$, where $c_{0}$ is independent of $x, y$, and $\theta$. Substituting into (6) gives

$$
f_{d}(x, y, \theta)=c_{0}+\epsilon \beta(x, y)-\epsilon^{2}\left(\frac{\partial \beta(x, y)}{\partial x} \cos \theta+\frac{\partial \beta(x, y)}{\partial y} \sin \theta\right)+\mathcal{O}\left(\epsilon^{3}\right)
$$

where $\beta(x, y)$ is an as-yet unknown function. Periodicity implies $\nabla^{2} \beta=0$, hence the nomenclature "diffusion solution". At quadrupole order, a coordinate-free representation is

$$
f_{d}(\mathbf{r}, \theta)=c_{0}+\epsilon \beta-\epsilon^{2} \nabla \beta \cdot \widehat{\mathbf{u}}+\frac{\epsilon^{3}}{4}\left(\partial_{i} \partial_{j} \beta\right) \hat{u}_{i} \hat{u}_{j}+\cdots
$$

An alternate way to identify $f_{d}$ as describing a diffusive process is to integrate it against $\hat{u}$, which results in a Fickian expression for the diffusive flux:

$$
\mathbf{J}_{d}(x, y)=\int \hat{u} f_{d}(x, y, \theta) d \theta=-\frac{\epsilon^{2}}{2} \nabla \rho_{d}(x, y)
$$

where $\rho_{d}=\int f_{d}(x, y, \theta) d \theta$ is the density. In contrast with the diffusion solution, the boundary layer does not possess a simple and general expression.

For a given problem, our strategy is to calculate the diffusion solution without recourse to the boundary layer or the full microscopic boundary condition. We accomplish this by making the following approximation: 
Diffusion approximation The diffusion solution $f_{d}(\boldsymbol{r}, \theta)$ carries all the particle flux normal to the boundary, that is,

$$
\int\left[\left.f(\mathbf{r}, \theta)\right|_{\mathbf{r} \in \partial S^{\prime}}-\left.f_{d}(\mathbf{r}, \theta)\right|_{\mathbf{r} \in \partial S^{\prime}}\right](\hat{u} \cdot \hat{n}) d \theta=0 ; \quad \rightarrow \quad \hat{n} \cdot \mathbf{J}=\hat{n} \cdot \mathbf{J}_{d}
$$

where $\hat{n}$ is the unit normal vector at a point on the boundary, and $f(\mathbf{r}, \theta)$ is the full solution to the problem. $\mathbf{J}$ and $\mathbf{J}_{d}$ correspond respectively to the fluxes associated with the full solution and the diffusion solution.

The motivation for this assumption begins with the fact that it is exact for the $1 \mathrm{~d}$ version of Eq. (4); see Refs. 73 and 74. Suppose now that the $1 \mathrm{~d}$ problem is understood as a reduction of the $2 \mathrm{~d}$ version in the presence of a symmetry, such as translational or rotational invariance. Then, one would expect Eq. (10) to hold approximately for a $2 \mathrm{~d}$ problem that only slightly breaks such a symmetry: such a problem is "nearly $1 \mathrm{~d}$ ". We will see later that the "nearly $1 \mathrm{~d}$ " qualifier corresponds to boundary conditions and domain constraints that are large compared with the microscopic length scale, in this case $\ell$.

The usefulness of the diffusion approximation is that it generates a well-posed boundary condition on $f_{d}$. To see this, note that Eq. (10) can also be written as

$$
\hat{n} \cdot \nabla \rho_{d}(x, y)=-\frac{2}{\epsilon^{2}} \int f(\mathbf{r}, \theta)(\hat{u} \cdot \hat{n}) d \theta=-\frac{2}{\epsilon^{2}}(\hat{n} \cdot \mathbf{J})
$$

where $\rho_{d}(x, y)=\int f_{d}(x, y, \theta) d \theta=$ constant $+2 \pi \epsilon \beta(x, y)$ is the density contribution from the diffusion solution. Since $\nabla^{2} \beta=0$, it follows that $\nabla^{2} \rho_{d}=0$ as well. Crucially, if the flux normal to the boundary is known - that is, $\hat{n} \cdot \mathbf{J}$ is given - then $\rho_{d}$ can be obtained (up to an additive constant) as the solution of the Laplace equation with Neumann boundary conditions. In turn, $\rho_{d}$ determines $f_{d}(x, y, \theta)$ in its entirety via Eq. (8).

\section{Benchmarking the framework: planar geometry}

As a first step, we validate our assertion that the diffusion approximation captures the bulk properties of the ABPs in the presence of boundaries. We do this by comparing the diffusion approximation with an exact solution to the planar $2 \mathrm{~d}$ problem. Specifically, we consider a system with a wall at $x=0$ and seek to solve for the steady state distribution $f(x, y, \theta)$ in the region $0<x<\infty$ and $-\infty<y<\infty$ with boundary conditions

$$
\begin{array}{ll}
f(0, y, \theta)=g(y, \theta) & \\
f(x, y, \theta) \rightarrow 0 & \text { as } x \rightarrow \infty
\end{array}
$$

\subsubsection{Separation of variables}

In the Cartesian coordinates relevant for this problem, Eq. (4) is separable. Taking $f=$ $\Gamma(x) \Xi(y) \Theta(\theta)$ leads to 


$$
\begin{aligned}
& \frac{d \Gamma}{d x}+\lambda \Gamma=0 ; \quad \frac{d \Xi}{d y}+v \Xi=0 \\
& \frac{d^{2} \Theta}{d \theta^{2}}+(\lambda \cos \theta+v \sin \theta) \Theta=0
\end{aligned}
$$

where $\lambda$ and $v$ are constants. To satisfy the general $y$-dependence of the boundary condition $x=0$, the functions $\Xi(y)$ need to be complete on $-\infty<y<\infty$. Thus, we take $v$ to be purely imaginary but otherwise unconstrained, writing $v=\mu i$, where $\mu$ is real. We hope that for each $\mu$, equations (14)-(15) admit a sequence of eigenvalues $\lambda_{k}(\mu)$ and eigenfunctions $\Theta_{k}(\theta, \mu)$ which are complete in $\theta$.

\subsubsection{Diffusion solutions}

Let us now consider the asymptotic solution in the bulk in the diffusion approximation formulated above,

$$
f_{d}(x, y, \theta)=c_{0}+\epsilon \beta(x, y)-\epsilon^{2}\left(\cos \theta \frac{\partial \beta(x, y)}{\partial x}+\sin \theta \frac{\partial \beta(x, y)}{\partial y}\right)+\mathcal{O}\left(\epsilon^{3}\right)
$$

where $\nabla^{2} \beta(x, y)=0$. We anticipate separable solutions with a $(x, y)$ dependence of the form $e^{c x} e^{ \pm i c y}$ where $c$ is a constant. This suggests taking $\lambda= \pm \mu$ in equations (14)-(15). Eq. (15) then becomes

$$
\frac{d^{2} \Theta}{d \theta^{2}} \pm \mu e^{ \pm i \theta} \Theta=0
$$

Making the change of variable $u=2 i( \pm \mu)^{\frac{1}{2}} e^{ \pm \frac{i \theta}{2}}$ this turns into

$$
u^{2} \frac{d^{2} \Theta}{d u^{2}}+u \frac{d \Theta}{d u}+u^{2} \Theta=0
$$

which is Bessel's equation with order 0 . The periodic boundary condition in $\theta$ implies $\Theta(u)=\Theta(-u)$, and thus the desired solution is the Bessel function of the first kind $J_{0}(u)$. In summary, the functions

$$
f_{d}(x, y, \theta, \mu)=e^{\mp \mu x} e^{-\mu i y} J_{0}\left(2 i( \pm \mu)^{\frac{1}{2}} e^{ \pm \frac{i \theta}{2}}\right)
$$

are solutions of (4) for any real $\mu$. A direct calculation from (8) shows that these are indeed the diffusion solutions.

\subsubsection{Boundary layer solutions}

Now, in order to construct the exact solution, we need to tackle the boundary layer, and this is difficult to calculate. Let us begin by making the following change of variables, $(\lambda, \mu)=$ ( $r \cos \gamma,-i r \sin \gamma$ ), where $\lambda, \mu, r$ are real and $\gamma$ is complex. Substitution into (15) gives

$$
\frac{d^{2} \Theta}{d \omega^{2}}+r \cos \omega \Theta=0
$$

where $\omega=\theta-\gamma$ and this equation needs to be solved with periodic boundary conditions. Eq. (20) is an eigenvalue equation of the indefinite Sturm-Liouville type that we studied in prior work [74]. It can be shown that there are an infinite number of eigenvalues which are discrete, real, and antisymmetric about 0 ; we index these with $k$ according to $\ldots r_{-2}<r_{-1}<r_{0}<r_{1}<r_{2}<\cdots$, where $r_{0}=0$. Thus, the spectrum of the 2-parameter problem (15) can be expressed as

$$
\begin{aligned}
& \lambda_{k}(\mu)=\operatorname{sgn}(k) \sqrt{\left|r_{k}\right|^{2}+\mu^{2}}, \quad k \in \mathbb{Z} /\{0\} \\
& \lambda_{0}^{ \pm}(\mu)= \pm|\mu|
\end{aligned}
$$

We denote the corresponding eigenfunctions as $\Theta_{k}(\theta, \mu)$ and $\Theta_{0}^{ \pm}(\theta, \mu)$. Using this transformation, the $\Theta_{k}(\theta, \mu)$ can be constructed through analytic continuation of the known eigenfunctions for $\mu=0$; see 
Ref. 75 for details. Like the $\mu=0$ case, the spectrum is discrete, real, and antisymmetric about 0 . Nevertheless, there are important differences. While the eigenvalues $\lambda_{k}(\mu)$ are real, the eigenfunctions $\Theta_{k}(\theta, \mu)$ are not. In addition, 0 is no longer an eigenvalue for nonzero $\mu$. Instead, the null space of the of the $\mu=0$ problem transforms into the space spanned by $\Theta_{0}^{ \pm}(\theta, \mu)$, which are the diffusion solutions from the previous section.

It is reasonable to expect that the $\Theta_{k}(\theta, \mu)$ for $\mu \neq 0$ satisfy full- and half-range completeness theorems analogous to those which hold for the $\mu=0$ eigenfunctions. Numerical evidence suggests that such theorems hold for the real part of the $\Theta_{k}$, denoted by $\Omega_{k}$ [75]:

$$
\Omega_{k}(\theta,|\mu|) \equiv \Re\left[\Theta_{k}(\theta, \mu)\right]=\frac{\Theta_{k}(\theta, \mu)+\Theta_{k}(\theta,-\mu)}{2}
$$

where we have used the fact that $\Theta_{k}(\theta,-\mu)$ is the complex conjugate of $\Theta_{k}(\theta, \mu)$. To formulate the completeness conjectures, let $\mathcal{H}$ be the Hilbert space associated with the inner product $\langle f, g\rangle=$ $\int_{-\pi}^{\pi} f(\theta) g(\theta)|\cos \theta| d \theta$, and let $\mathcal{H}_{ \pm}$be the respective subspaces where $\cos \theta>0$ or $\cos \theta<0$. Then, we conjecture the following:

- Full-range completeness - For real and non-zero $\mu$, any real-valued function in $\mathcal{H}$ can be expanded in terms of the (also real-valued) functions $\left\{\Omega_{k}(\theta,|\mu|), \Omega_{0}^{ \pm}(\theta,|\mu|)\right\}$, where $k \in \mathbb{Z} /\{0\}$.

- Half-range completeness - For real and non-zero $\mu$, any real-valued function in $\mathcal{H}_{+}$can be expanded in terms of the (also real-valued) functions $\left\{\Omega_{k}(\theta,|\mu|), \Omega_{0}^{+}(\theta,|\mu|)\right\}$, where $k>0$. Similarly, any real-valued function in $\mathcal{H}_{-}$can be expanded in terms of $\left\{\Omega_{k}(\theta,|\mu|), \Omega_{0}^{-}(\theta,|\mu|)\right\}$, where $k<0$.

Interestingly, the imaginary part of the $\Theta_{k}$ does not appear to share the completeness properties of the real part. As explained in the next section, however, both real and imaginary parts are required for solving the full boundary value problem. In anticipation of this, we introduce similar shorthand notation as for the real part:

$$
\Upsilon_{k}(\theta,|\mu|) \equiv \mathfrak{J}\left[\Theta_{k}(\theta, \mu)\right]=\frac{\Theta_{k}(\theta, \mu)-\Theta_{k}(\theta,-\mu)}{2 i}
$$

\subsubsection{Full solution of a boundary value problem}

To construct the full solution, our strategy is to express $f(x, y, \theta)$ as a linear combination of separable solutions from above, varying $\lambda$ and $\mu$ over the range of allowed values. In view of the asymptotic condition on $f, \lambda$ must be positive, which restricts us to half of the spectrum. We then have

$$
f(x, y, \theta)=\int_{-\infty}^{\infty} d \mu\left[\sum_{k \geq 0} a_{k}(\mu) e^{-\lambda_{k}(\mu) x} \Theta_{k}(\theta, \mu)\right] e^{-i \mu y}
$$

where the $k=0$ mode corresponds to the sign of $\mu$, i.e. $\Theta_{0}=\Theta_{0}^{\operatorname{sgn}(\mu)}$ and $\lambda_{0}(\mu)=\operatorname{sgn}(\mu) \mu$. Since the boundary condition $g(y, \theta)$ is real-valued, it is convenient to separate everything into real and imaginary parts. For compactness, we do not write the explicit $\mu$ and $\theta$ arguments on the $\Omega_{k}$ and $\Upsilon_{k}$ (each has arguments $+\theta$ and $|\mu|)$. Then, 

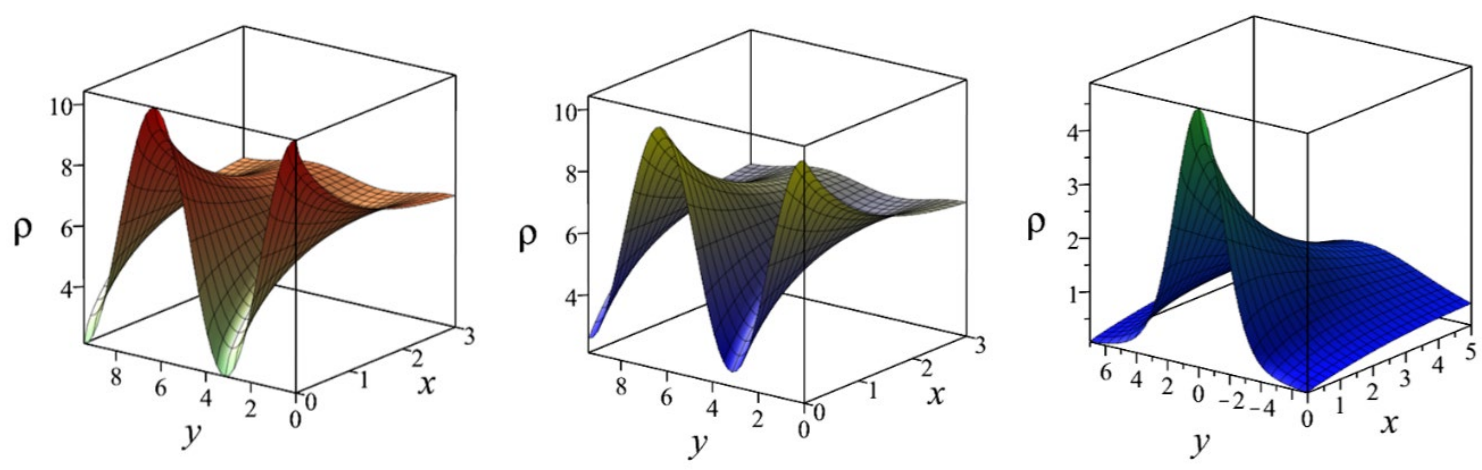

Figure 2: Densities for the planar problem with two example boundary conditions. Left and middle: $f(0, y, \theta)=2+\cos y$, with the left displaying the density $\rho(x, y)$ computed using (26) and the least-squares method and the middle showing the density contribution from the diffusion solution, $\rho_{d}(x, y) \simeq$ $3.693 \times e^{-x} \cos y$. The two qualitatively agree, validating the diffusion approximation. Right: $f(0, y, \theta)=$ $e^{-y^{2} / 4}$, density computed using the least squares method. Comparing to the left panel, this illustrates the strong influence of the boundary on the bulk solution.

$$
\begin{aligned}
& f(x, y, \theta)=\int_{0}^{\infty} d \mu \sum_{k \geq 0}\left\{+\Re\left[a_{k}(\mu)\right] \Omega_{k}-\Im\left[a_{k}(\mu)\right] \Upsilon_{k}+\Re\left[a_{k}(-\mu)\right] \Omega_{k}+\Im\left[a_{k}(-\mu)\right] \Upsilon_{k}\right\} e^{-\lambda_{k} x} \cos \mu y \\
& \quad+\int_{0}^{\infty} d \mu \sum_{k \geq 0}\left\{+\Re\left[a_{k}(\mu)\right] \Upsilon_{k}+\Im\left[a_{k}(\mu)\right] \Omega_{k}+\Re\left[a_{k}(-\mu)\right] \Upsilon_{k}-\Im\left[a_{k}(-\mu)\right] \Omega_{k}\right\} e^{-\lambda_{k} x} \sin \mu y \\
& +i \int_{0}^{\infty} d \mu \sum_{k \geq 0}\left\{+\Re\left[a_{k}(\mu)\right] \Upsilon_{k}+\Im\left[a_{k}(\mu)\right] \Omega_{k}-\Re\left[a_{k}(-\mu)\right] \Upsilon_{k}+\Im\left[a_{k}(-\mu)\right] \Omega_{k}\right\} e^{-\lambda_{k} x} \cos \mu y \\
& +i \int_{0}^{\infty} d \mu \sum_{k \geq 0}\left\{-\Re\left[a_{k}(\mu)\right] \Omega_{k}+\Im\left[a_{k}(\mu)\right] \Upsilon_{k}+\Re\left[a_{k}(-\mu)\right] \Omega_{k}+\Im\left[a_{k}(-\mu)\right] \Upsilon_{k}\right\} e^{-\lambda_{k} x} \sin \mu y
\end{aligned}
$$

where the $k=0$ mode is the positive one, i.e. $\Omega_{0}=\Omega_{0}^{+}$and $\Upsilon_{0}=\Upsilon_{0}^{+}$. By comparison, the first boundary condition can similarly be expressed as a Fourier integral:

$$
f(0, y, \theta)=g(y, \theta)=\int_{0}^{\infty} d \mu q(\mu, \theta) \cos \mu y+\int_{0}^{\infty} d \mu p(\mu, \theta) \sin \mu y, \quad \cos \theta>0
$$

The problem is solved if, for given $\mu>0$, we can choose $a_{k}(\mu)$ such that the quantities in brackets in Eq. (26) match the respective functions $q(\theta, \mu)$ and $p(\theta, \mu)$. This results in four independent equations with four independent sets of coefficients: $\Re\left[a_{k}( \pm \mu)\right]$ and $\mathfrak{I}\left[a_{k}( \pm \mu)\right]$. We solve these equations using a least-squares technique which appears to depend on the completeness of the $\Omega_{k}$ but also requires use of the $\Upsilon_{k}$. The validity of the procedure is supported by good numerical convergence on a variety of test functions [75]. 

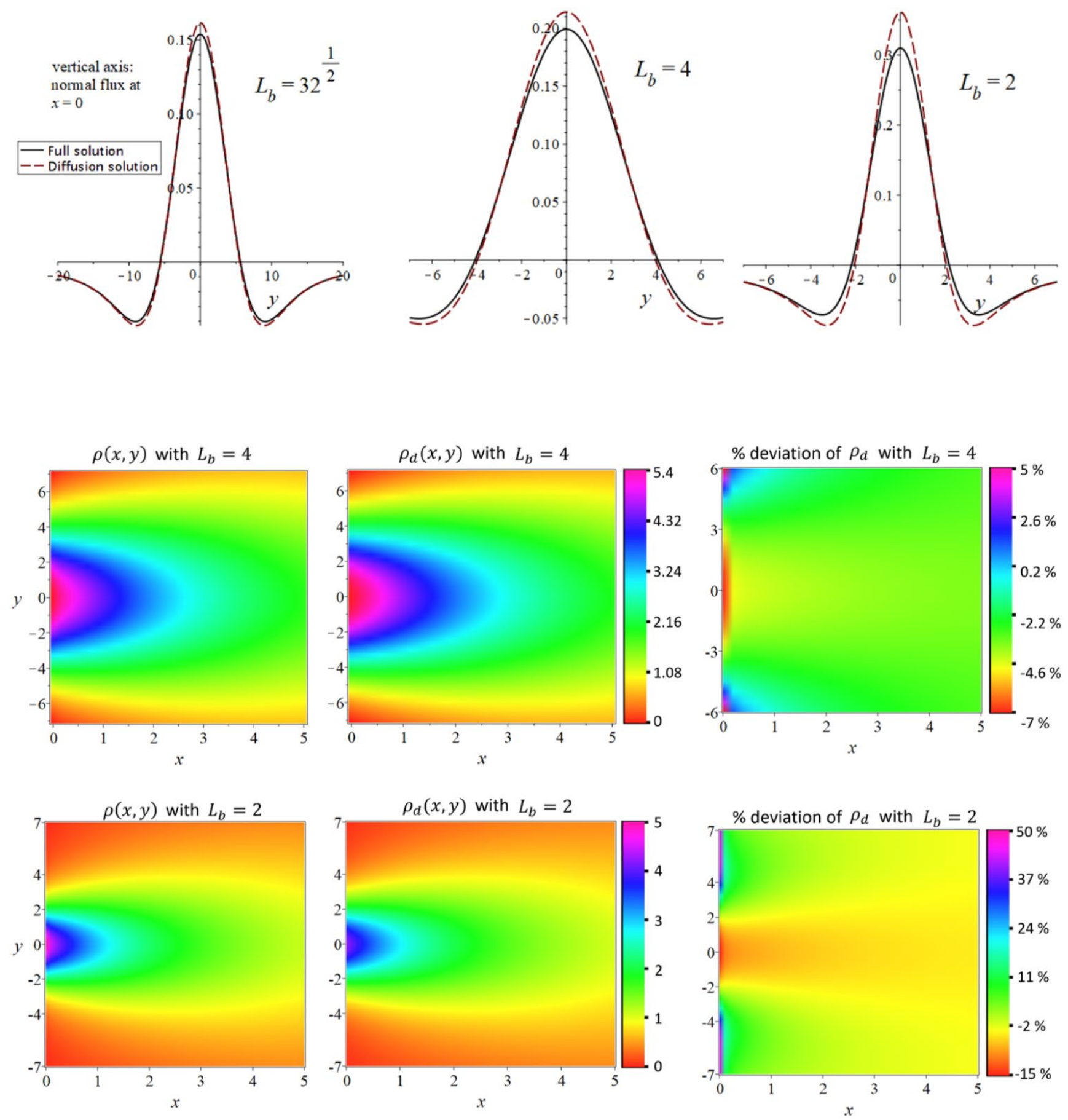

Figure 3: Tests of the diffusion approximation in the planar geometry with boundary condition $f(0, y, \theta)=$ $e^{-\left(\mathrm{y} / L_{b}\right)^{2}}$. The top row shows the normal flux at $(x, y)=(0,0)$. The diffusion solution indeed carries most of the flux for $L_{b}>1$, with the fraction increasing with $L_{b}$. This supports our claim that the diffusion approximation is valid so long as the length characterizing the variation of the boundary data ( $L_{b}$ in this case) is large compared with $\ell$. In the bottom two rows, we compare the density calculated in the diffusion approximation $\left(\rho_{d}\right)$ with the exact density $(\rho)$. As expected, the approximation increases in accuracy as $L_{b}$ becomes large compared with $\ell$. 
Here we show results for the cases $g(y, \theta)=2+\cos y$ and $g(y, \theta)=e^{-y^{2} / 4}$. The first boundary condition could model ABPs in an infinite channel whose walls have periodically varying absorption energies, whereas the second describes perfectly absorbing walls in the presence of a steady particle source centered around $y=0$. The densities corresponding to these scenarios are shown in Fig. 2. As the first boundary condition involves only a single mode of the set of $y$ eigenfunctions, the diffusion solution is easy to write down:

$$
f_{d}(x, y, \theta) \simeq 1.176 \cdot e^{-x}\left(\Re\left[J_{0}\left(2 i e^{\frac{i \theta}{2}}\right)\right] \cos y+\mathfrak{J}\left[J_{0}\left(2 i e^{\frac{i \theta}{2}}\right)\right] \sin y\right)
$$

The density is just $\rho_{d}(x, y) \simeq 3.693 \cdot e^{-x} \cos y$, which compares well with the exact solution (Fig. 3).

\subsubsection{Application of the diffusion approximation}

While formally exact, the separation of variables approach has to be implemented numerically and may not work well in problems with discontinuous boundary conditions. By contrast, the diffusion approximation defined in section 3 trades the exactness of this approach for a substantial simplification in complexity: given basic physical assumptions, one can obtain an approximate analytic expression for the solution in the bulk without needing to solve for the boundary layer. Here we test this approximation for the planar geometry. Importantly, knowing the exact solution in the form (25), we can analytically separate the diffusion solution from the boundary layer and test the diffusion approximation directly, which is not possible for other geometries considered later. Specifically, we compute the the normal flux $J_{x}$ for the boundary condition $g(y, \theta)=e^{-\frac{y^{2}}{L_{b}^{2}}}$. Fig. 3 compares $J_{x}$ in the diffusion approximation with the exact result, for various $L_{b}$. The two quantities coincide with high accuracy for $L_{b} \gg 1$, and even when $L_{b} \sim 1$, the agreement is at least qualitative.

\section{Illustration 1: circular geometry}

\subsection{Problem statement}

We define polar coordinates $(r, \alpha)$ by $x=r \cos \alpha$ and $y=r \sin \alpha$. Applying this transformation to Eq. (4) gives

$$
\cos (\alpha-\theta) \frac{\partial f}{\partial r}-\frac{\sin (\alpha-\theta)}{r} \frac{\partial f}{\partial \alpha}=\frac{\partial^{2} f}{\partial \theta^{2}}
$$

In a geometry which is radially symmetric, $f$ depends on $\alpha$ and $\theta$ only through the combination $\phi \equiv$ $\alpha-\theta$. Then, (29) simplies to

$$
\cos \phi \frac{\partial f}{\partial r}-\frac{\sin \phi}{r} \frac{\partial f}{\partial \phi}=\frac{\partial^{2} f}{\partial \phi^{2}}
$$

where $-\pi<\phi<\pi$. Perhaps surprisingly, neither of these equations is separable. More generally, we have not found Eq. (4) to be separable in any spatial coordinates besides rectangular. Thus, the diffusion approximation is essential for analyzing even simple curvilinear geometries, since the boundary layer can no longer be accessed via a separation of variables. 
In this section, we solve the above equations with a variety of boundary conditions. First, we consider an annular region $R_{1}<r<R_{2}$, in which case boundary conditions take the form

$$
\begin{aligned}
& f\left(R_{1}, \alpha, \theta\right)=B_{1}(\alpha, \theta) \quad \text { where } \cos \phi>0 \\
& f\left(R_{2}, \alpha, \theta\right)=B_{2}(\alpha, \theta) \quad \text { where } \cos \phi<0
\end{aligned}
$$

We consider both spherically symmetric and non-symmetric boundary data, focusing on the qualitative differences between the solutions in either case. Second, we consider the infinite exterior region $r>$ $R_{1}$, in which case the second boundary condition is replaced by $\lim _{r \rightarrow \infty} f(r, \alpha, \theta)=$ constant.

\subsection{Diffusion solution}

The diffusion solution in the spherically symmetric case of (30) is easy to calculate:

$$
\begin{aligned}
f_{d}(r, \phi) & =a+b\left(\ln r-\sum_{m=1}^{\infty} \frac{1}{m ! m} \frac{\cos m \phi}{r^{m}}\right) \\
& \equiv a+b g(r, \phi)
\end{aligned}
$$

where $a$ and $b$ are constants. Note that since (30) is effectively a $1 \mathrm{~d}$ problem, the most general diffusion solution contains only two undetermined constants $[26,5]$. In the absence of spherical symmetry, the problem is of course more complicated and in this case, to quadrupole order, we have

$$
f_{d}(r, \alpha, \theta)=c_{0}+\beta-\frac{\partial \beta}{\partial r} \cos \phi+\frac{1}{r} \frac{\partial \beta}{\partial \alpha} \sin \phi+\frac{\partial^{2} \beta}{\partial r^{2}} \cos 2 \phi+\frac{1}{r} \frac{\partial^{2} \beta}{\partial r \partial \alpha} \sin 2 \phi+\cdots
$$

where, as mentioned above, $\beta(r, \alpha)$ solves $\nabla^{2} \beta=0$. With this in mind, we introduce the multipole expansion

$$
\beta(r, \alpha)=-\frac{\boldsymbol{p} \cdot \hat{r}}{r}+\frac{Q_{i j} \hat{r}_{i} \hat{r}_{j}}{r^{2}}+\mathcal{O}\left(\frac{1}{r^{3}}\right)
$$

where the constant term has been absorbed into $c_{0}$, and $\boldsymbol{p}$ and $\mathbf{Q}$ are determined from the boundary conditions on $\beta(r, \alpha)$.

\subsection{Symmetric circular geometry}

In the case of spherical symmetry, the diffusion approximation is exact. The reason can be understood by integrating 30 with respect to $\phi$, which gives

$$
\hat{r} \cdot \mathbf{J}=\int f \cos \phi d \phi=\frac{\text { constant }}{r}
$$

Comparing with (33), we see that the diffusion solution also has this property. On the other hand, the boundary layer part of the solution decays exponentially with $r$ away from the boundary, and therefore cannot contribute to the overall flux without violating (36). Thus, the diffusion approximation is exact,

and the constant $b$ in (34) is equal to $-\frac{\hat{r} \cdot \mathbf{J}}{\pi}$. Fig. 4 compares the density $\rho_{d}(r)$ obtained in this way with a finite-element solution from PDE2D, verifying its accuracy in the bulk of the region. 

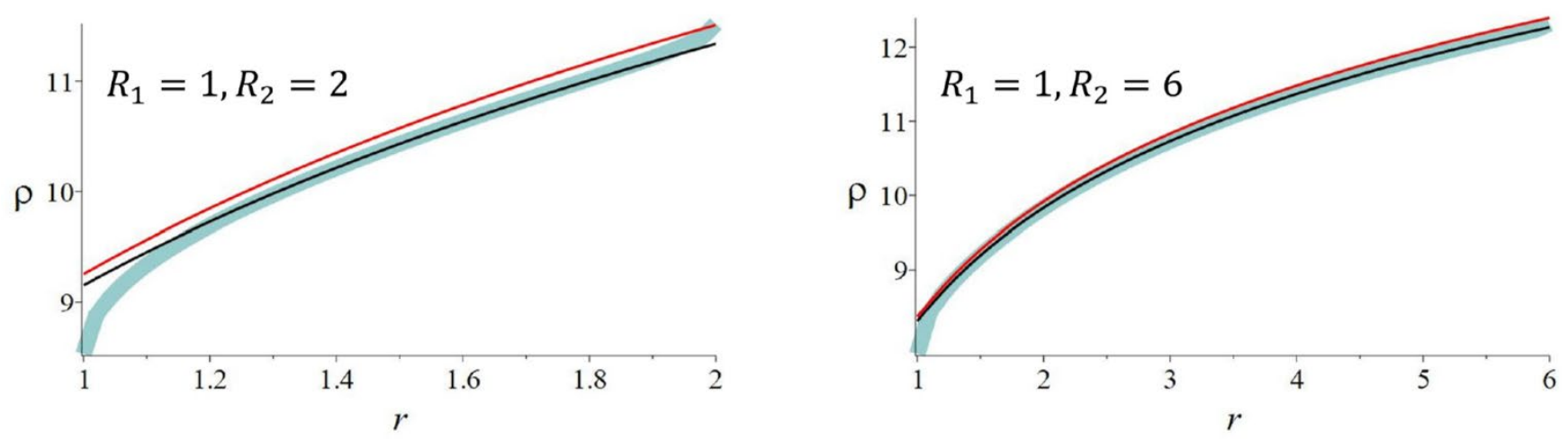

Numerical solution $\quad-\rho_{d}$, exact

$\rho_{d}$, Eqs. 38 and 39

Figure 4: Density $\rho$ for the symmetric annular problem (boundary conditions (31)-(32) with $B_{1}=1, B_{2}=2$ ).

An alternate (though approximate) approach estimates the solution directly in terms of the boundary functions $B_{1}$ and $B_{2}$, and therefore does not require prior knowledge of $J$. The idea is to choose $a$ and $b$ such that the boundary conditions are satisfied "in the mean" with respect to weight functions $\cos \phi$ and $\cos ^{2} \phi$ :

$$
\begin{aligned}
& \int_{\cos \phi>0}\left[B_{1}(\phi)-f_{d}\left(R_{1}, \phi\right)\right] \cos \phi d \phi+\int_{\cos \phi<0}\left[B_{2}(\phi)-f_{d}\left(R_{2}, \phi\right)\right] \cos \phi d \phi=0 \\
& \int_{\cos \phi>0}\left[B_{1}(\phi)-f_{d}\left(R_{1}, \phi\right)\right] \cos ^{2} \phi d \phi+\int_{\cos \phi<0}\left[B_{2}(\phi)-f_{d}\left(R_{2}, \phi\right)\right] \cos ^{2} \phi d \phi=0
\end{aligned}
$$

In a 1d planar geometry, this approximation is the first term in a convergent iterative scheme [73], and so we expect it to be valid for large $R_{1}$ and $R_{2}-R_{1}$. Comparison with numerics confirms this expectation (Fig. 4).

\subsection{Asymmetric circular geometry}

In contrast with a spherically symmetric geometry, asymmetric boundary conditions can generate currents and long-range density variations. In a system of ABPs, one way to achieve this is with a fixed, spherical inclusion which attracts and binds particles on one side but is purely repulsive on the other side. Then, particles could escape only from one side of the sphere. For concreteness, suppose that the side with binding interactions is the left side, i.e. where $x<0$. Then the boundary conditions would be

$$
\begin{aligned}
& f(R, \alpha, \theta)=\left\{\begin{array}{lr}
g_{+}(\alpha, \theta) & \text { where } \cos \alpha>0 \text { and } \cos (\theta-\alpha)>0 \\
0 & \text { where } \cos \alpha<0 \text { and } \cos (\theta-\alpha)>0 \\
\text { unspecifed } & \text { else }
\end{array}\right. \\
& \lim _{r \rightarrow \infty} f(r, \alpha, \theta)=c_{0}=\text { constant }
\end{aligned}
$$



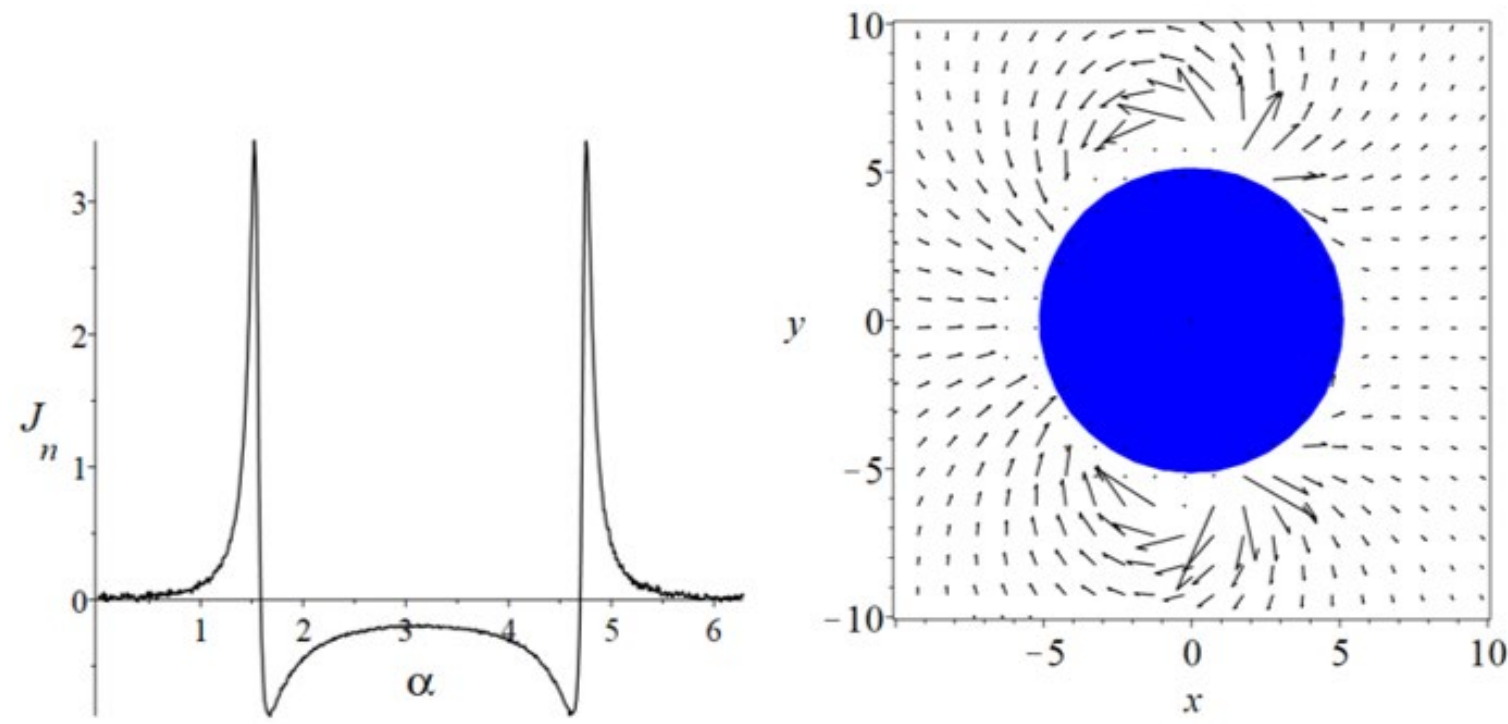

Figure 5: In a closed system of ABPs in steady state, particle-surface interactions that break spherical symmetry are sufficient to induce current loops that begin and end on different points of the boundary. This figure shows the results for one such system, in which the particle-surface interactions with a spherical inclusion are derived

from a continuous, time-independent potential energy function, (see Eq. 40, and a detailed explanation in appendix A). Qualitatively, this interaction potential has the effect of binding particles on the left side of the boundary and releasing them on the right side. The left plot shows the normal flux $J_{\mathrm{n}}$ at the boundary (black points) as a function of the angle $\alpha$ with respect to the $x$-axis, confirming that particles can only escape from the

right side of the sphere. The right plot shows the flux field as a vector plot, with length of the arrows proportional to the value of the flux at the point. (Flux vectors above a certain magnitude were excluded to improve visualization.)
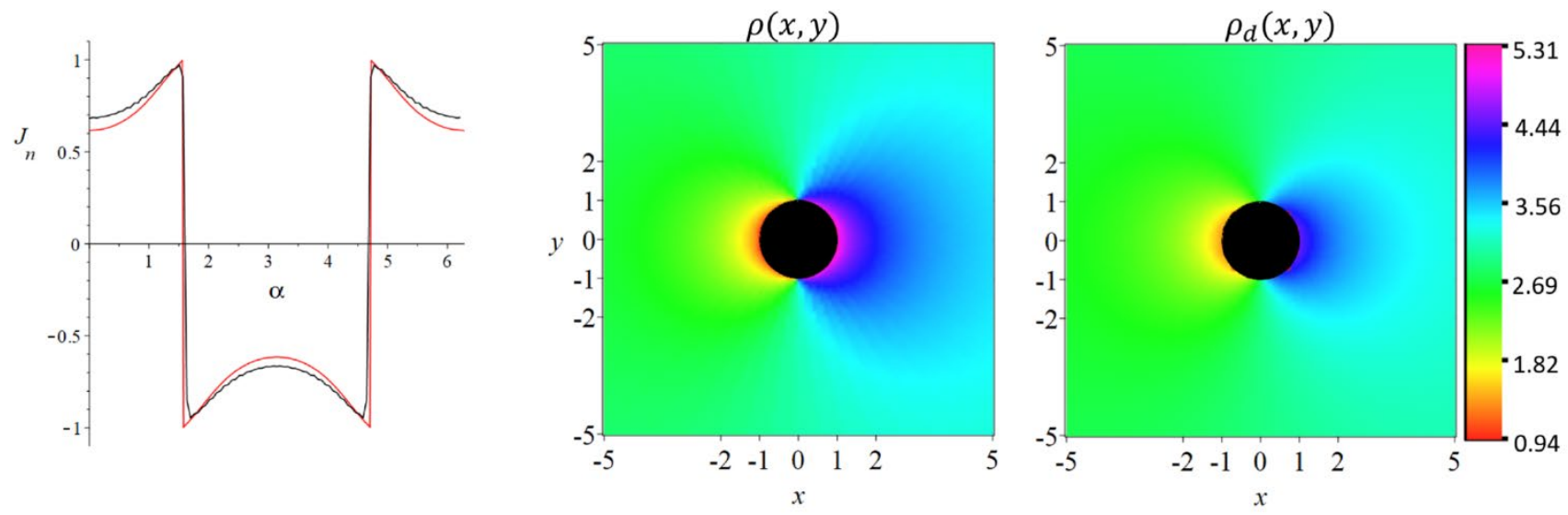

Figure 6: Tests of our analytical predictions for boundary conditions (40)-(41) with $R=1$ and $g_{+}=1$. The left plot compares the boundary normal flux $J_{\mathrm{n}}$ from the finite element solution (black points) with the analytical result (red curve) from the locally planar analysis in appendix $B$. The other plots compare the particle density in the diffusion approximation ( $\rho_{d}$, middle plot) with the finite-element solution ( $\rho$, left plot). The agreement is good given that the analytical approximations have no free parameters. 
such that $g_{+}(\alpha, \theta)$ gives the "exit distribution" of particles leaving the right side of the sphere. Since the particles are bound to the left side of the sphere, the corresponding exit distribution there is 0 . Note that $g_{+}(\alpha, \theta)$ covers only half of the $(\alpha, \theta)$ domain. In fact, on the left side of the sphere, $f(R, \alpha, \theta)$ is nonzero on the complement of the interval where it is 0 (the "entrance distribution). However, only the exit distribution in Eq. (40) is required to form a well-posed boundary-value problem.

While the exit distribution is exactly 0 on the left side of the sphere, the corresponding distribution $g_{+}(\alpha, \theta)$ on the right side depends on the statistics of particle trajectories in the vicinity of the sphere, and requires a more detailed analysis that we do not attempt here. For example, one would need to calculate the probability that a trajectory starting on the left side of the sphere would eventually depart the right side at given $(\alpha, \theta)$.

Instead, we test the validity of the diffusion approximation independent of the particle-surface kinetics by taking $g_{+}$to be exactly 1 . In the general case, the diffusion approximation for the density, $\rho_{d}(r, \alpha)$, is

$$
\begin{aligned}
& \rho_{d}(r, \alpha)=2 \pi c_{0}+\sum_{m=1}^{\infty}\left(a_{m} \frac{\cos m \alpha}{r^{m}}+b_{m} \frac{\sin m \alpha}{r^{m}}\right) \\
& {\left[\begin{array}{l}
a_{m} \\
b_{m}
\end{array}\right]=\frac{2}{\pi} \frac{R^{m+1}}{m} \int_{-\pi}^{\pi} J_{\mathrm{n}}(\alpha)\left[\begin{array}{c}
\cos m \alpha \\
\sin m \alpha
\end{array}\right] d \alpha}
\end{aligned}
$$

where $J_{\mathrm{n}}(\alpha) \equiv \hat{n} \cdot \mathbf{J}(R, \alpha)$ is the normal flux at the boundary. Ref [75] works out a strategy for estimating $J_{\mathrm{n}}$ from $g_{+}$. The idea is to treat the boundary as locally planar and use the machinery from section 4.0.3 to compute the solution close to the boundary (see Appendix B). Fig. 6 compares $J_{\mathrm{n}}(\alpha)$ calculated in this way for $R=1$ with with the numerical solution from PDE2D. The agreement is surprisingly good considering that $R$ is equal to the persistence length $\ell=1$, which a priori would call into question the accuracy of the locally planar approximation. For larger $R$, the approximation is even better [75]. In particular, we find the simple expression $J_{\mathrm{n}}(\alpha) \approx(R \cos \alpha)^{-1}$ to be accurate for $R \gg 1$, which in the multipole expansion (36) implies a dipole moment

$$
\mathbf{p}=\left(a_{1}, b_{1}\right)=(4 R, 0), \quad(R \gg 1)
$$

Fig. 6 compares the resulting $\rho_{d}$ with the finite-element solution. If we exclude the region within a persistence length of the boundary (white annulus), the maximum percent deviation of $\rho_{d}$ is about $5 \%$. Thus, the diffusion approximation works very well in a nontrivial curvilinear geometry, where an exact solution by separation of variables is no longer possible. 


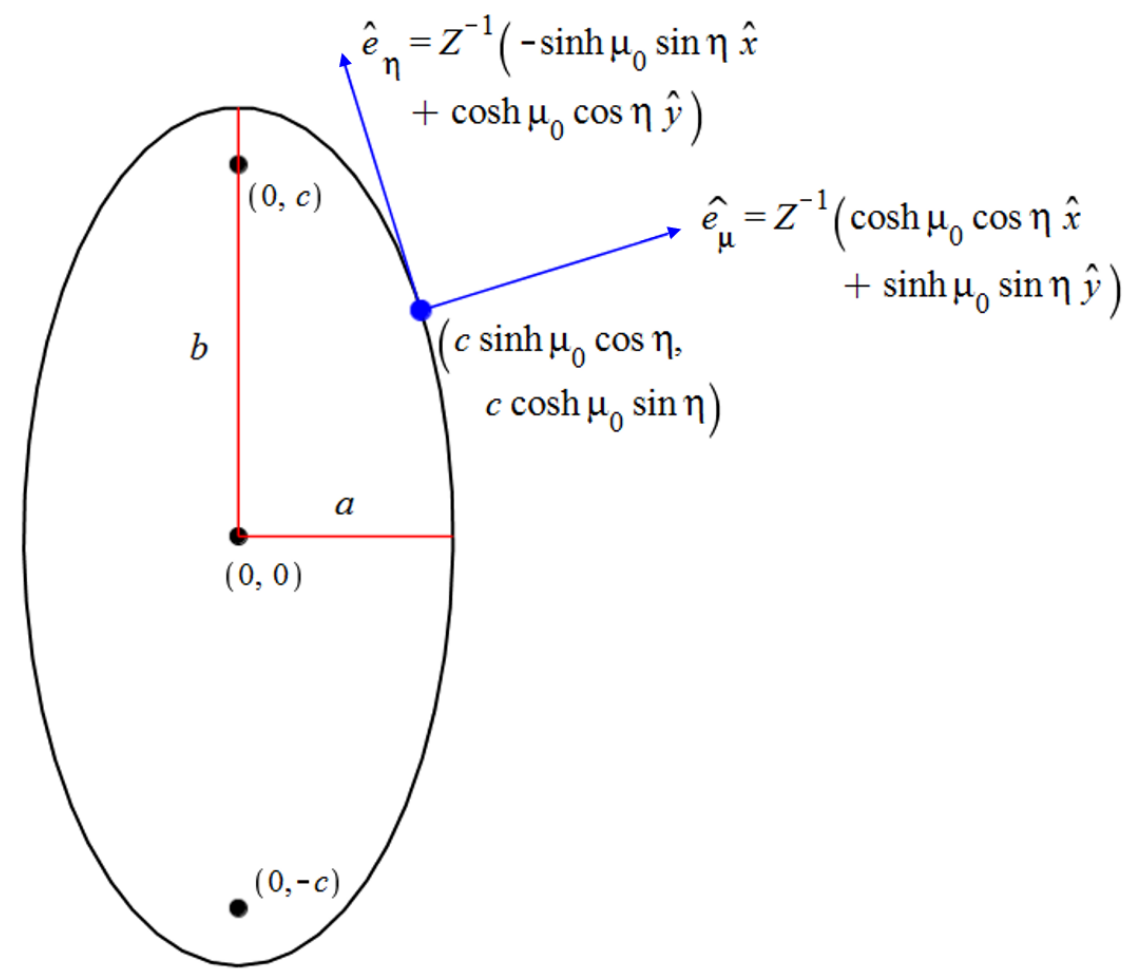

Figure 7: Elliptic geometry. We define elliptic coordinates $(\mu, \eta)$ by $x=c \sinh \mu \cos \eta$ and $y=c \cosh \mu \sin \eta$, where $c=\sqrt{b^{2}-a^{2}}$. These are orthogonal coordinates, and the constant $\mu$ surfaces are confocal ellipses. In particular, $\mu=\mu_{0} \equiv \operatorname{arccosh}\left(\frac{b}{c}\right)$ defines the ellipse depicted on the right. The blue arrows are the unit tangent and normal vectors at the point $\left(\mu_{0}, \eta\right)$; the unit normal vector is the same as the coordinate vector $\hat{e}_{\mu}$, and similarly the tangent vector is $\hat{e}_{\eta} \cdot Z^{-1}=\left(\cosh ^{2} \mu_{0} \cos ^{2} \eta+\sinh ^{2} \mu_{0} \sin ^{2} \eta\right)^{-1 / 2}$ is a normalization factor.

\section{Illustration 2: elliptic geometry}

In the polar geometry, an asymmetry in the particle-surface interaction is required to generate long-range flow; otherwise, the net particle current is everywhere 0 and density decays exponentially away from the boundary. In particular, a purely repulsive spherical inclusion does not generate currents. In this section, we turn to an elliptic geometry, in which the requisite asymmetry is present in the shape of the boundary itself. Experimentally, such geometries would allow one to engineer long-range flows without having to tune particle-surface interactions.

\subsection{Problem setup}

Fig. 7 shows the setup: we take our boundary to be a vertically oriented ellipse $E$ with semimajor axis $a$, semiminor axis $b$, and foci at $(0, \pm c)$ where $c \equiv \sqrt{b^{2}-a^{2}}$. We define elliptic coordinates $(\mu, \eta)$,

$$
\left\{\begin{array}{l}
x=c \sinh \mu \cos \eta \\
y=c \cosh \mu \sin \eta
\end{array}\right.
$$



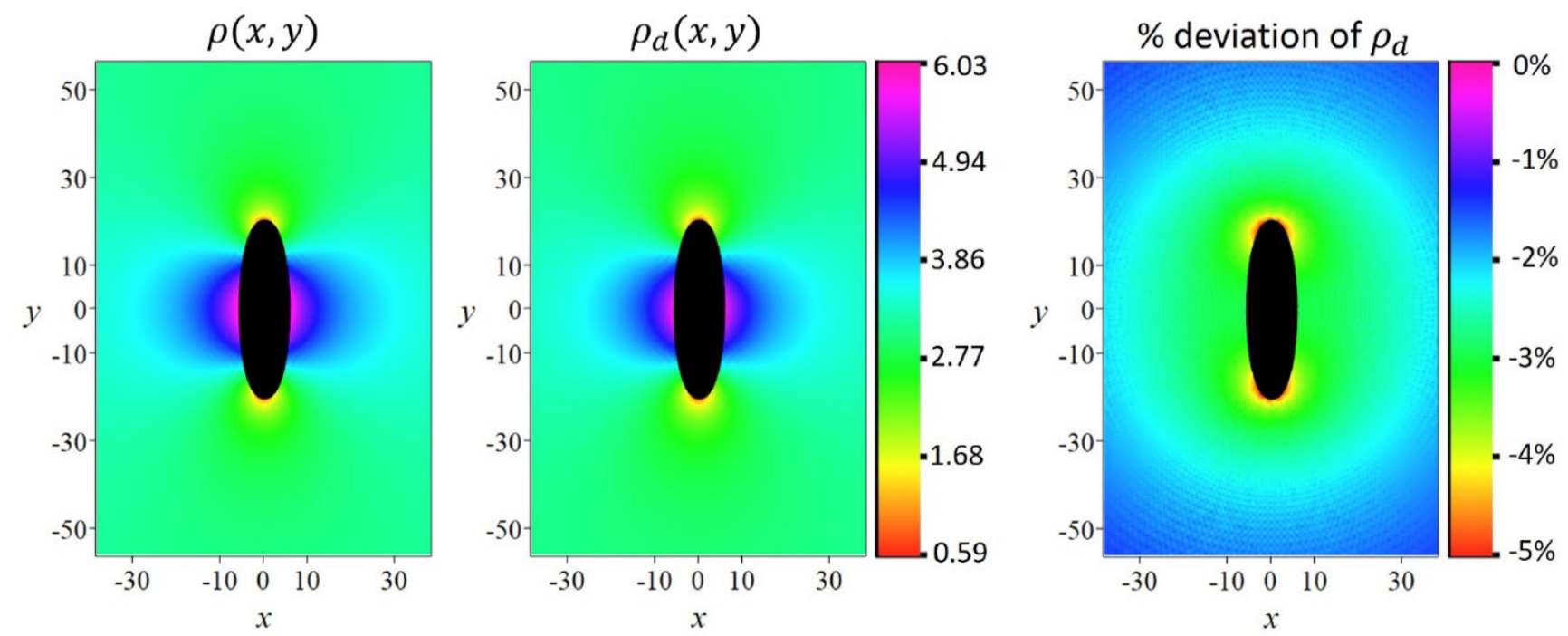

Figure 8: Density profiles corresponding to the boundary condition $f\left(\mu_{0}, \eta, \theta\right)=\cos ^{2} \eta$ and $f \rightarrow$ constant as $\mu \rightarrow \infty$. The $\mu=\mu_{0}$ coordinate surface defines an ellipse (black) with semimajor axis $b=20 \ell$ and semiminor axis $a=5 \ell$. The left and middle plots show the density from PDE2D and the diffusion approximation, respectively. The rightmost plot shows the percent deviation, which is systematic but small.

such that $E$ is defined by the constant- $\mu$ surface $\mu=\mu_{0} \equiv \operatorname{arccosh}\left(\frac{b}{c}\right)$. The diffusion solution can be written in terms of $\mu$ and $\eta$ using the general expression

$$
f_{d}(\mathbf{r}, \theta)=c_{0}+\beta(\mathbf{r})-\epsilon^{2} \nabla \beta(\mathbf{r}) \cdot \hat{u}+\frac{\epsilon^{3}}{4}(\nabla \nabla \beta(\mathbf{r}))_{i j} \hat{u}_{i} \hat{u}_{j}+\cdots
$$

where $\nabla^{2} \beta(\mathbf{r})=0$. Conveniently, the Laplacian is just $\nabla^{2}=\partial_{\mu}^{2}+\partial_{v}^{2}$, so that $\beta(\mu, \eta)$ can be expanded as

$$
\beta(\mu, \eta)=\sum_{m=1}^{\infty} e^{-m \mu}\left(a_{m} \cos m \eta+b_{m} \sin m \eta\right)
$$

In the diffusion approximation, the boundary condition is

$$
\left.\left(\hat{e}_{\mu} \cdot \nabla \beta(\mu, \eta)\right)\right|_{\mu=\mu_{0}}=-J_{\mathrm{n}}(\eta) \rightarrow \frac{1}{c\left(\sinh ^{2} \mu_{0}+\cos ^{2} \eta\right)^{\frac{1}{2}}} \frac{\partial \beta}{\partial \mu}=-J_{\mathrm{n}}(\eta)
$$

where $\hat{e}_{\mu}$ is the $\mu$-coordinate vector (which is the same as the unit normal vector) and $J_{\mathrm{n}}(\eta)$ is the normal flux. Thus,

$$
a_{m}=\frac{c}{m \pi} e^{m \mu_{0}} \int_{0}^{2 \pi}(\cos m \eta)\left(\sinh ^{2} \mu_{0}+\cos ^{2} \eta\right)^{\frac{1}{2}} J_{\mathrm{n}}(\eta) d \eta
$$

and similarly for $b_{m}$. Transforming from $(\mu, \eta)$ to $(x, y)$ gives

where

$$
\beta(\mu, \eta)=\frac{\boldsymbol{p} \cdot \hat{r}}{r}+\frac{Q_{i j} \hat{r}_{i} \hat{r}_{j}-\left(\frac{1}{2}\right) \operatorname{Tr} \mathbf{Q}}{r^{2}}+\mathcal{O}\left(\frac{1}{r^{3}}\right)
$$




$$
\boldsymbol{p}=\frac{c}{2}\left(a_{1}, b_{1}\right) ; \quad \mathbf{Q}=\frac{c^{2}}{4}\left(\begin{array}{ll}
a_{2} & b_{2} \\
b_{2} & a_{2}
\end{array}\right)
$$

Fig. 8 compares the diffusion solution with PDE2D for $a=10, b=50$, and the boundary condition

$$
\begin{gathered}
f\left(\mu_{0}, \eta, \theta\right)=\cos ^{2} \eta \\
\lim _{\mu \rightarrow \infty} f(\mu, \eta, \theta)=\mathrm{constant}
\end{gathered}
$$

The agreement is on par with what we saw for the circular geometry. Of course, for a physical system the boundary conditions must be derived self-consistently from the underlying equations of motion, (1)(2). To apply the diffusion approximation, in particular, we need to know the boundary normal flux. Here we present a kinetic argument which approximates this quantity in the case of a long and thin ellipse ( $a / b$ small), and moderate to large persistence length $\ell$.

The idea is that in these circumstances, a particle which adsorbs onto the surface tends to drive itself toward the ends of the ellipse before it significantly changes its orientation. This implies that most particles leave the surface in the vicinity of the points $(0, \pm b)$. By contrast, if $\ell$ were much smaller than $b$, then particles would have sufficient time to reorient themselves and leave before they travel too far from where they got adsorbed. To know the net flux, we also have to estimate the incoming flux of particles. Here we simply take this to be constant along the surface: the idea is that a particle returning to the surface has spent enough time in the bulk to forget where it left the surface. Again, we expect this to be a reasonable approximation for sufficiently large $\ell$.

\subsection{Test of the diffusion approximation}

From the above, we are led to the following expression for the boundary normal flux:

$$
\begin{aligned}
& J_{\mathrm{n}}(\eta)=\frac{\chi}{2 a}\left[\delta\left(\eta-\frac{\pi}{2}\right)+\delta\left(\eta-\frac{3 \pi}{2}\right)-\frac{2 a}{c I_{0}}\right] \\
& \text { where } I_{0} \equiv \int_{0}^{2 \pi}\left(\sinh ^{2} \mu_{0}+\cos ^{2} \eta\right)^{\frac{1}{2}} d \eta
\end{aligned}
$$

The $\delta$-functions model the outgoing flux due to particles leaving near $(0, \pm b)$, and the constant offset $\left(\frac{1}{\pi}\right)$ accounts for the incoming flux, its value chosen so that the net flux integrated along the surface is 0 . The function $F(\eta)$ expresses these two contributions up to an undetermined constant $\chi$, which has units of inverse time. Physically, $\chi$ quantifies the total number of particles impinging on (or leaving) the surface in unit time. With this interpretation, a rough guess is that $\chi \approx 2 c_{0} \mathcal{A} v_{0}, v_{0}$ being the selfpropulsion velocity, $c_{0}$ the (constant) value of $f$ at infinity, and $\mathcal{A}$ the surface area.

Fig. 9 compares this prediction with simulation for $a=0.25 \ell, b=\ell$, and particle radius $0.1 \ell$. The measured normal flux reflects the general shape of $F(\eta)$, showing peaks near $\cos \eta=0$ and a constant offset. The predicted density $\rho_{d}$ using Eq. (54) also compares well with simulation: most of the error is concentrated in the near field, particularly around $( \pm a, 0)$. 

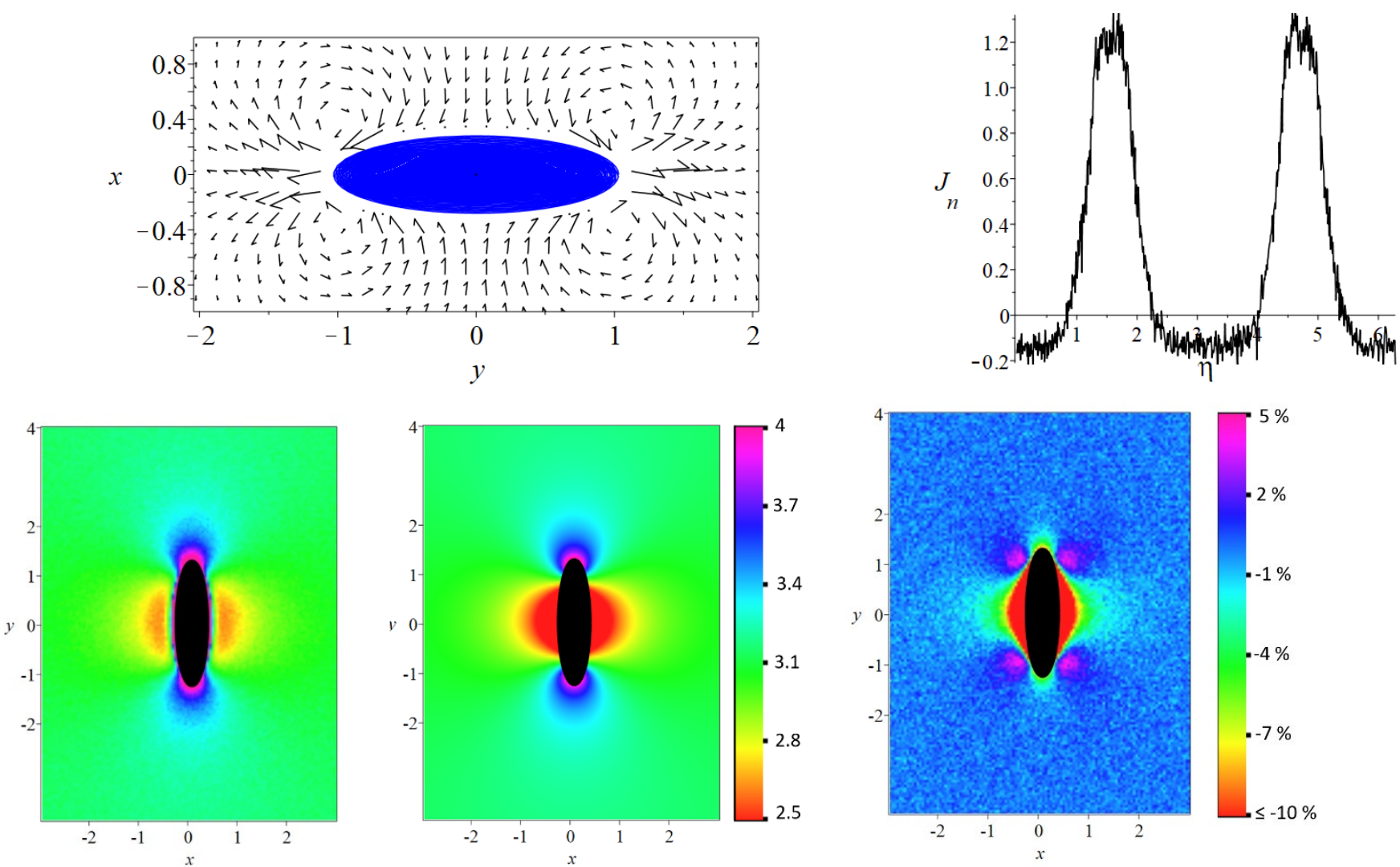

Figure 9: Flux field and density for a bath of ABPs in contact with a fixed, purely repulsive ellipse with semimajor axis $b=\ell$ and semiminor axis $a=0.25 \ell$. The radius of an ABP is $0.1 \ell$. The top two plots show the flux field and normal flux $J_{\mathrm{n}}$ obtained from simulation of the particle dynamics (1)-(2). The bottom left plot shows the density field from simulations. The bottom middle plot shows the diffusion approximation using the boundary condition (54). The agreement is fairly good considering the simplifications leading up to (54).

The predicted dipole moment is 0 , and the quadrupole moment is $Q_{12}=Q_{21}$,

$$
Q_{11}=Q_{22}=-\frac{\mathcal{A}}{8 \pi}(a+b)^{2}\left(1+\frac{I_{2}}{I_{0}}\right)
$$

where

$$
I_{m} \equiv \int_{0}^{2 \pi} \cos m \eta\left(\sinh ^{2} \mu_{0}+\cos ^{2} \eta\right)^{\frac{1}{2}} d \eta
$$

In the limit $b \gg a$, this becomes $Q_{11}=Q_{22} \approx(2 \pi)^{-1}(a+b)^{2} \mathcal{A}$. For the problem in Fig. 9 , the numerical value is $Q_{11} \simeq-0.42$.

A caveat is that the predicted flux, Eq. (54), is larger than the measured flux by roughly an order of magnitude. Despite this discrepancy, using (54) as the boundary condition on the diffusion solution ends up giving a more accurate prediction than using the measured flux. A likely explanation is that the fraction of the flux contained in the exact diffusion solution is greater than 1 , the excess being balanced by the boundary layer piece of the solution. Then, the better agreement of (54) is due to a cancellation of two errors: the first overestimating the flux and the second underestimating the fraction of flux contained in the diffusion solution. Such situations are expected to occur when $\ell$ is the same order of 
magnitude as the length scale of the boundary conditions, as is the case here. Nevertheless, our results suggest that the diffusion approximation may give a qualitative understanding of the steady state even in these cases.

\section{Summary}

Our goal has been to develop an analytical framework to understand ABP steady states in the presence of boundaries. A key simplification that enables this effort is the diffusion approximation, Eq. (10), which says that the asymptotic ("diffusion") solution of (4) carries all the particle flux. Our results from sections 4-6 demonstrate the validity of this approximation when variations of the boundary data are small compared with the persistence length $\ell$ (which we set to 1 in the majority of our analysis). More generally, the diffusion approximation provides a map between violations of detailed balance at the boundary and corresponding mass fluxes in the bulk. This mapping could be a useful design principle for nonequilibrium steady states in general: by tuning particle-boundary interactions or boundary geometry, one could achieve desired properties of the bulk steady state.

There are obvious extensions of our methods. First, while we have matched the asymptotic solution with a single physical quantity at the boundary - the normal flux - one can envision a similar procedure involving additional physical quantities, for instance the nematic order parameter $Q_{i j}=$ $\int \hat{u}_{i} \hat{u}_{j} f(\boldsymbol{r}, \theta) d \theta$ or other moments with respect to $\theta$. For comparison, a similar moment-based approach for the classical Boltzmann equation is Grad's 13-moment system and extensions [28, 29, 68]. On the other hand, the diffusion solution is already determined by the normal flux, so additional solutions would have to be included to avoid overdetermining the problem. For the planar problem considered in Section 4, one could, for instance, include the next-order separable solution, $e^{-\lambda_{1} x} e^{i \mu y} \Theta_{1}$. However, it is not clear that such an extension would generate new qualitative insights. Moreover, successful implementation would require prior knowledge of additional physical quantities at the boundary, which may not be practical.

The extension of our framework to higher-dimensional problems could be useful, as a way to mitigate the exponential increase in computational burden of a direct numerical simulation. Even in cases where higher accuracy is desired than provided by the diffusion approximation, the general form of the asymptotic solution could be used to accelerate numerical algorithms [33]. Along these lines, a study of long-range flow fields in $3 d$ ABP systems would be interesting.

Finally, the extension to time-dependent problems would be interesting. The characteristics of the long-time approach to diffusion have lately been considered in the active matter context [61, 41, 59, $4,71]$, and an asymptotic approach such as ours could provide additional insight. For the ABP model, a possible starting point is to scale Eq. (4) with $\epsilon$ as

$$
\epsilon^{2} \partial_{t} f+\epsilon\left(\cos \theta \partial_{x} f+\sin \theta \partial_{y} f\right)=\partial_{\theta}^{2} f
$$

Then, substituting $f \sim f_{0}+\epsilon f_{1}+\cdots$ gives $f=$ constant $+\beta(x, y, t)+\mathcal{O}(\epsilon)$, where $\beta$ solves $\partial_{t} \beta=(1 / 4) \nabla^{2} \beta$. Thus, in this scaling, the long-time evolution is indeed diffusive. Since the inital value problem does not involve a complicated half-range analysis, connecting the diffusion solution with initial conditions should pose fewer technical difficulties than the analagous boundary value problem (for e.g., see Malvagi1991 and Grad1963). Thus, our work, while directly providing an analytical handle on ABP 
steady states, can serve as a springboard to generalize tools developed in the context of classical fluids to problems of topical interest in active matter.

While preparing this article, we learned of a preprint [22] that also treats bulk-boundary correspondence and long-range effects in active colloid steady states, and arrives at similar conclusions regarding the density and current profiles near a planar wall. Their treatment of interparticle interactions is an interesting and nontrivial extension to our analysis, which is restricted to dilute systems. On the other hand, our work goes beyond theirs in that we calculate exactly the boundary layer in the planar problem, as well as treat more general curvilinear geometries. Moreover, by formulating the microscopic transport equation as a well-posed boundary value problem, we provide a systematic procedure for constructing and validating bulk-boundary correspondence principles such as the diffusion approximation in section 3 .

Acknowledgments. We acknowledge support from the Brandeis Center for Bioinspired Soft Materials, an NSF MRSEC, DMR-1420382 (CGW, AB, MFH), NSF-MRSEC-2011486 (AB, MFH) , NSF DMR1149266 and BSF-2014279 (CGW and AB), and DMR-1855914 (MFH). CGW also acknowledges support from the Heising-Simons Foundation, the Simons Foundation, and National Science Foundation Grant No. NSF PHY-1748958. Computational resources were provided by the NSF through XSEDE computing resources (MCB090163) and the Brandeis HPCC which is partially supported by the Brandeis MRSEC and NSF OAC-1920147. We also thank Granville Sewell for assistance with PDE2D.

\section{Appendix A: Numerical methods}

For the particle-based simulations, we numerically integrate (1) and (2) using a stochastic Euler algorithm. Our simulations are ideal for GPU architectures since the code is trivial to parallelize (particles are non-interacting), and so we wrote a CUDA implementation alongside $\mathrm{C}++$ code for CPUs. In most cases we use timestep $\Delta t=\mathcal{O}\left(10^{-5} D_{r}^{-1}\right)$. In the $\mathrm{C}++$ code, however, we use $10 \Delta t$ as timestep far from the boundary, where the solution is slowly varying, which results in a substantial speed-up. We take statistics at intervals of $D_{r}^{-1}$, beginning at a threshold time of around $10^{4} D_{r}^{-1}$. All simulations use $D_{t}=$ $0.001 \ell^{2} D_{r}$, except for the ellipse problem from section 6 , where we use $D_{t}=0.0001 \ell^{2} D_{r}$. The reason for a smaller value is to ensure the width $\delta$ of the diffusive boundary layer scales appropriately with the dimensions of the ellipse.

For the circular geometry (section 5.4), we use the particle-surface interaction potential

$$
\begin{aligned}
& V(r, \alpha)=\frac{\tanh (100 R-100 r)+1}{10}\left[\tanh \left(5 \alpha-\frac{5 \pi}{2}\right)+\tanh \left(\frac{15 \pi}{2}-5 \alpha\right)-2\right] \\
& +\frac{1}{10}\left(r+\frac{1}{19(r-R+1)^{19}}\right)-\frac{191}{190}[1-\mathrm{H}(R-r)]
\end{aligned}
$$

which is continuous in the region of interest. Here $H(z)$ is the Heaviside function. Roughly speaking, the potential gives rise to a "ratchet" along the surface of the circle. Where $x<0$ particles experience a strong but short-ranged force in the direction of $-\hat{r}$. By contrast, fixing $r$ and varying $\alpha$ from the left hemisphere to the right, particles experience a weaker but longer-range force in the direction of $-\hat{\alpha}$, i.e., pointing towards the left hemisphere. The asymmetry between the radial and angular components 
forms a ratchet that rectifies the motion of the ABPs in agreement with previous work on self-propelled particles in ratchet potentials [55]. The net effect is that particles tend to be bound on the left side of the sphere and released on the right.

The interactions for the elliptic geometry are defined as follows. For the repulsive force, $\hat{e}_{\eta} \cdot \boldsymbol{F}_{r}=$ 0 and

$$
\frac{\hat{e}_{\mu} \cdot F_{r}}{\ell D_{r}}= \begin{cases}(10 s)^{-40}-1 & 0<s \leq 0.1 \\ 0 & s>1\end{cases}
$$

where $s$ is the distance from an ellipse with semiminor and semimajor axes $a$ and $b$. While we would like to identify effective semimajor and semiminor axes induced by $\boldsymbol{F}_{r}$, these are not well-defined because the constant-distance surfaces, along which $\hat{e}_{\mu} \cdot \boldsymbol{F}_{r}$ is constant, are not ellipses. However, since the range of the force is small compared with the major- and minor-axes, this discrepancy is small. Indeed, a numerical calculation shows that effective values $a_{\mathrm{eff}}=a+0.1 \ell$ and $b_{\mathrm{eff}}=b+0.1 \ell$ define an ellipse which very nearly coincides with the $s=\ell$ surface.

We use the Fortran-based software PDE2D2 for our finite element computations. For solving 3dimensional problems like (4), it uses a collocation method with tricubic Hermite basis functions [62, 63]. In our analysis, we use 20 gridlines in the $\theta$ variable, 50 in the angular coordinate $\alpha$ or $\eta$, and at least 90 in the radial coordinate $r$ or $\mu$.

\section{Appendix B: Locally planar approximation}

Here we illustrate the type of "locally planar" approximation that can be used to estimate the boundary normal flux. We use the same setup as from section 5.4, that is, a circular geometry with boundary data

$$
\begin{aligned}
& f(R, \alpha, \theta)= \begin{cases}1 & \text { where } \cos \alpha>0 \text { and } \cos (\theta-\alpha)>0 \\
0 & \text { where } \cos \alpha<0 \text { and } \cos (\theta-\alpha)>0 \\
\text { unspecifed } & \text { else }\end{cases} \\
& \lim _{r \rightarrow \infty} f(r, \alpha, \theta)=c_{0}=\text { constant }
\end{aligned}
$$

to be solved on $R<r<\infty, 0<\alpha<2 \pi,-\pi<\theta<\pi$. The constant $c_{0}$ is constrained by the boundary condition at $r=R$. In a planar problem, $c_{0}$ would correspond to the constant Fourier mode of (60), which in the present case gives $c_{0}=\frac{1}{2}$. For the range of $R$-values considered here, the actual value is slightly smaller and carries a weak $R$-dependence. For instance, $c_{0} \approx 0.466$ for $R=1$ and 0.452 for $R=$ 5 . However, consistent with the level of approximation adopted elsewhere in this section, we simply take $c_{0}=\frac{1}{2}$. Then, it is convenient to subtract $c_{0}$ from the overall solution and solve the transformed problem

$$
\begin{aligned}
& f(R, \alpha, \theta)= \begin{cases}+\frac{1}{2} & \text { where } \cos \alpha>0 \text { and } \cos (\theta-\alpha)>0 \\
-\frac{1}{2} & \text { where } \cos \alpha<0 \text { and } \cos (\theta-\alpha)>0 \\
\text { unspecifed } & \text { else }\end{cases} \\
& \lim _{r \rightarrow \infty} f(r, \alpha, \theta)=0
\end{aligned}
$$

${ }^{2}$ www.pde2d.com 

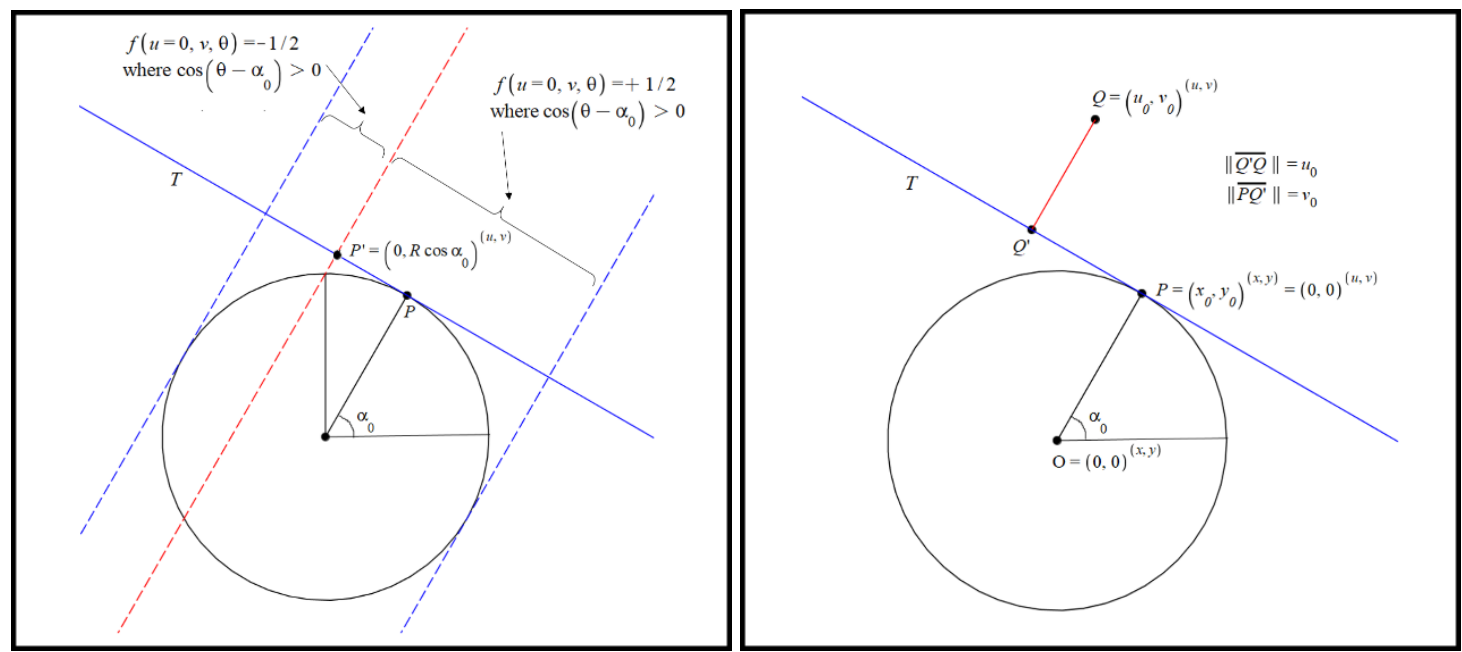

Figure 10: Ingredients in the locally planar approximation. The left panel illustrates the $(u, v)$ coordinate system corresponding to a point $P$ on the boundary. $u$ and $v$ are the horizontal and vertical coordinates in a planar half-space problem bounded on the left by the tangent line $T(u=0)$. The right panel shows how we approximate the boundary conditions at $u=0$ by "projecting" onto $T$ the boundary conditions (64), which sit on the surface of the sphere. The idea is that particles retain their approximate angular distribution as they leave

the sphere and pass through $T$.

The locally planar approximation is illustrated in Fig. 10. Consider a point $P$ on the boundary, with Cartesian coordinates $\left(x_{0}, y_{0}\right)$ and polar coordinates $\left(R, \alpha_{0}\right)$, and tangent line $T$. We can define an infinite half-space problem if we consider $T$ as defining a planar boundary: if $f(r, \alpha, \theta)$ is known along the entirety of $T$, then we can use the methods of section 4 to solve for $f(r, \alpha, \theta)$ on the infinite half space outside of $T$ (on the side opposite the circle).

Towards this end, for a point $Q$ above $T$, let $u$ be the perpendicular distance from $T$ and $v$ be the parallel distance from $P$. We denote these coordinates with superscript $(u, v)$ such that $Q=(u, v)^{(u, v)}$ and $P=(0,0)^{(u, v)}$. In terms of these coordinates, suppose we know $f(u=0, v, \theta)$ on $-\infty<v<\infty$ and where $\cos \left(\theta-\alpha_{0}\right)>0$. Then, $f(u, v, \theta)$ solves an infinite half-space problem on $u>0$ of the type solved analytically in section 4 , with boundary conditions $f(u=0, v, \theta)=g(v, \theta)$ defined on $-\infty<$ $v<\infty, \cos \left(\theta-\alpha_{0}\right)>0$. It remains to determine $g(v, \theta)$.

Suppose we can satisfy ourselves with solving for $f$ only very close to $P$. This is certainly true if our goal is to determine the boundary normal flux at $P$. Being close to $P$ means that the form of $g(u, \theta)$ for large $|v|$ influences the solution much less than its behavior near $v=0$, so we can get away with some approximations in the former region. In this spirit, the locally planar approximation "projects" the boundary conditions at $r=R$ onto $T$. For the point $P$, for instance, we would take

$$
\begin{aligned}
& \left.g(v, \theta)\right|_{\cos \left(\theta-\alpha_{0}\right)>0}= \begin{cases}+\frac{1}{2} & -R<u<R \cos \alpha_{0} \\
-\frac{1}{2} & R \cos \alpha_{0}<u<R \\
0 & |u|>R\end{cases} \\
& \operatorname{Lim}_{r \rightarrow \infty} f(r, \alpha, \theta)=a_{0}=\text { constant }
\end{aligned}
$$

Though less accurate for large $|v|$, this approximation will generate accurate boundary conditions near 


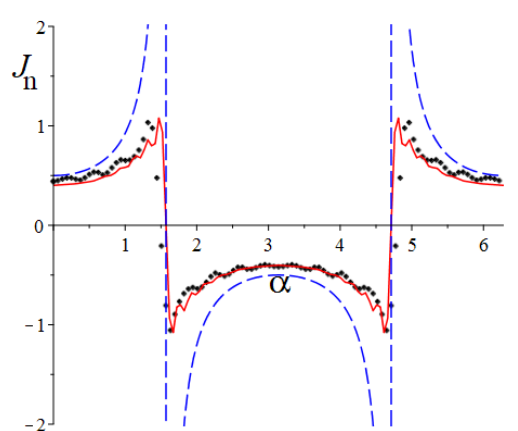

$\mathrm{R}=2$

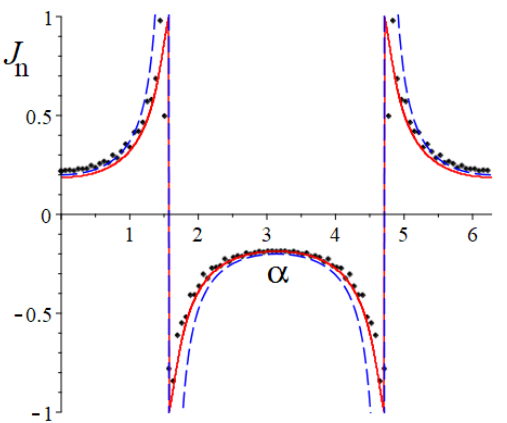

$\mathrm{R}=5$

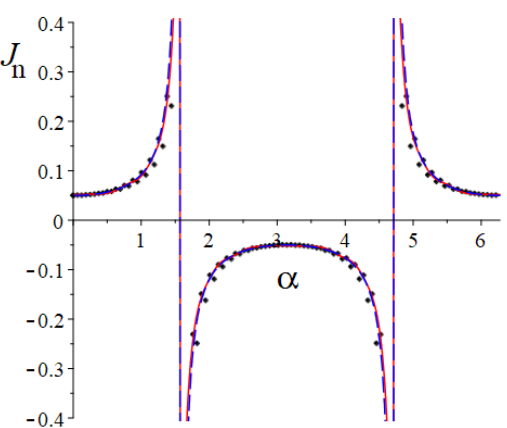

$\mathrm{R}=20$

Figure 11: Some tests of the locally planar approximation for $R=2,5,20$. The black diamonds show the normal flux $J_{\mathrm{n}}(\alpha)$ evaluated numerically using PDE2D. The solid red curve shows the locally planar approximation described in appendix 9 , and the blue dashed curve shows the function $(R \cos \alpha)^{-1}$. The accuracy of the approximations increases with $R$, but is good even for $R=2$.

$P$, and the exact boundary condition at $P$ itself. Thus, resulting solution $f(r, \alpha, \theta)$ is also expected to be accurate near $P$.

Fig. 11 tests the approximation for several $R$, comparing the calculated boundary normal flux $J_{\mathrm{n}}(R, \alpha)$ with the finite-element solution. The agreement is surprisingly good over a range of $R$ values. If $R$ is large and $\cos \alpha$ not too close to 0 , then $J_{\mathrm{n}}(R, \alpha)$ is $J_{\mathrm{n}}(R, \alpha) \approx(R \cos \alpha)^{-1}$, which is also shown in Fig. 11.

\section{References}

[1] L. Angelani, C. Maggi, M. L. Bernardini, A. Rizzo, and R. Di Leonardo. Effective interactions between colloidal particles suspended in a bath of swimming cells. Phys. Rev. Lett., 107:138302, Sep 2011.

[2] Alessandro Attanasi, Andrea Cavagna, Lorenzo Del Castello, Irene Giardina, Tomas $S$ Grigera, Asja Jelić, Stefania Melillo, Leonardo Parisi, Oliver Pohl, Edward Shen, and Massimiliano Viale. Information transfer and behavioural inertia in starling flocks. Nat. Phys., 10:691, 2014.

[3] Yongjoo Baek, Alexandre P. Solon, Xinpeng Xu, Nikolai Nikola, and Yariv Kafri. Generic longrange interactions between passive bodies in an active fluid. Phys. Rev. Lett., 120:058002, Jan 2018.

[4] Urna Basu, Satya N. Majumdar, Alberto Rosso, and Grégory Schehr. Long-time position 
distribution of an active brownian particle in two dimensions. Phys. Rev. E, 100(6), December 2019.

[5] R. Beals. Indefinite sturm-liouville problems and half-range completeness. J. Differential Equations, 56(3):391 - 407, 1985.

[6] Ch. Becco, N. Vandewalle, J. Delcourt, and P. Poncin. Experimental evidences of a structural and dynamical transition in fish school. Physica A, 367:487-493, 2006.

[7] Clemens Bechinger, Roberto Di Leonardo, Hartmut Löwen, Charles Reichhardt, Giorgio Volpe, and Giovanni Volpe. Active particles in complex and crowded environments. Rev. Mod. Phys., 88:045006, Nov 2016.

[8] Antoine Bricard, Jean-Baptiste Caussin, Nicolas Desreumaux, Olivier Dauchot, and Denis Bartolo. Emergence of macroscopic directed motion in populations of motile colloids. Nature, 503:95-98, Nov 2013.

[9] DorÃlson Silva CambuÃ and Alexandre Rosas. Density induced transition in a school of fish. Physica A, 391(15):3908 - 3914, 2012.

[10] Kenneth M. Case and Paul F. Zweifel. Linear Transport Theory. Addison-Wesley, 1967.

[11] Michael E. Cates and Julien Tailleur. Motility-induced phase separation. Annu. Rev. Condens. Matter Phys., 6(1):219-244, 2015.

[12] Jordana Cepelewicz. How swarming insects act like fluids. Quanta Magazine, July 2019.

[13] Carlo Cercignani. Plane couette flow according to the method of elementary solutions. J. Math. Anal. Appl., 11:93-101, 1965.

[14] Carlo Cercignani. Plane poiseuille flow according to the method of elementary solutions. J. Math. Anal. Appl., 12(2):254 - 262, 1965.

[15] Carlo Cercignani. Mathematical Methods in Kinetic Theory. Springer US, 1969. 
[16] Sydney Chapman and T.G. Cowling. The Mathematical Theory of Non-uniform Gases. Cambridge University Press, 3rd edition, 1970.

[17] Olivier Dauchot and Vincent Démery. Dynamics of a self-propelled particle in a harmonic trap. Phys. Rev. Lett., 122(6), February 2019.

[18] Michael A. Day. The no-slip condition of fluid dynamics. Erkenntnis, 33(3):285-296, November 1990.

[19] A. Deblais, T. Barois, T. Guerin, P.H. Delville, R. Vaudaine, J.S. Lintuvuori, J.F. Boudet, J.C. Baret, and $\mathrm{H}$. Kellay. Boundaries control collective dynamics of inertial self-propelled robots. Phys. Rev. Lett., 120(18), May 2018.

[20] Julien Deseigne, Sebastien Leonard, Olivier Dauchot, and Hugues Chate. Vibrated polar disks: spontaneous motion, binary collisions, and collective dynamics. Soft Matter, 8:5629-5639, 2012.

[21] Christopher Dombrowski, Luis Cisneros, Sunita Chatkaew, Raymond E. Goldstein, and John O. Kessler. Self-concentration and large-scale coherence in bacterial dynamics. Phys. Rev. Lett., 93:098103, Aug 2004.

[22] Ydan Ben Dor, Sunghan Ro, Yariv Kafri, Mehran Kardar, and Julien Tailleur. The farreaching influence of boundaries on shaping bulk behavior of active matter, 2021.

[23] Jens Elgeti and Gerhard Gompper. Wall accumulation of self-propelled spheres. EPL (Europhysics Letters), 101(4):48003, 2013.

[24] Barath Ezhilan, Roberto Alonso-Matilla, and David Saintillan. On the distribution and swim pressure of run-and-tumble particles in confinement. J. Fluid Mech., 781, September 2015.

[25] Yaouen Fily and M. Cristina Marchetti. Athermal Phase Separation of Self-Propelled Particles with No Alignment. Phys. Rev. Lett., 108:235702, 2012.

[26] Nathaniel J. Fisch and Martin D. Kruskal. Separating variables in two-way diffusion 
equations. J. Math. Phys., 21(4):740-750, 1980.

[27] Étienne Fodor and M. Cristina Marchetti. The statistical physics of active matter: From self-catalytic colloids to living cells. Physica A, 504:106-120, August 2018.

[28] Harold Grad. On the kinetic theory of rarefied gases. Commun. Pure Appl. Math., 2:325, 1949.

[29] Harold Grad. Principles of the kinetic theory of gases. In S. Fluegge, editor, Thermodynamik der Gase / Thermodynamics of Gases, pages 205-294. Springer-Verlag, 1986.

[30] J. Harder, S. A. Mallory, C. Tung, C. Valeriani, and A. Cacciuto. The role of particle shape in active depletion. J. Chem. Phys., 141(19):194901, November 2014.

[31] Kevin Hartnett. Smart swarms seek new ways to cooperate. Quanta Magazine, Feb 2018.

[32] G. F. Hubmer and U. M. Titulaer. The kinetic boundary layer for the linearized boltzmann equation around an absorbing sphere. J. Stat. Phys., 59(1):441-459, Apr 1990.

[33] Shi Jin. Efficient asymptotic-preserving (AP) schemes for some multiscale kinetic equations. SIAM Journal on Scientific Computing, 21(2):441-454, January 1999.

[34] G. Junot, G. Briand, R. Ledesma-Alonso, and O. Dauchot. Active versus passive hard disks against a membrane: Mechanical pressure and instability. Phys. Rev. Lett., 119:028002, Jul 2017.

[35] A J Kainz and U M Titulaer. The analytic structure of the stationary kinetic boundary layer for brownian particles near an absorbing wall. J. Phys. A, 24(19):4677-4695, oct 1991.

[36] A J Kainz and U M Titulaer. The structure of the stationary kinetic boundary layer for the linear BGK equation. J. Phys. A, 25(11):3189-3203, jun 1992.

[37] Andreas Kaiser, Anton Peshkov, Andrey Sokolov, Borge ten Hagen, Hartmut Löwen, and Igor S. Aranson. Transport powered by bacterial turbulence. Phys. Rev. Lett., 112:158101, Apr 2014. 
[38] Chiu Fan Lee. Active particles under confinement: aggregation at the wall and gradient formation inside a channel. New J. Phys., 15(5):055007, 2013.

[39] L. R. Leite, D. Lucena, F. Q. Potiguar, and W. P. Ferreira. Depletion forces on circular and elliptical obstacles induced by active matter. Phys. Rev. E., 94(6), December 2016.

[40] Ke Li, Fuchen Guo, Xiaolin Zhou, Xianghong Wang, Linli He, and Linxi Zhang. An attractionrepulsion transition of force on two asymmetric wedges induced by active particles. Scientific Reports, 10(1), July 2020.

[41] Kanaya Malakar, V Jemseena, Anupam Kundu, K Vijay Kumar, Sanjib Sabhapandit, Satya N Majumdar, S Redner, and Abhishek Dhar. Steady state, relaxation and first-passage properties of a run-and-tumble particle in one-dimension. J. Stat. Mech., 2018(4):043215, apr 2018.

[42] M. C. Marchetti, J. F. Joanny, S. Ramaswamy, T. B. Liverpool, J. Prost, Madan Rao, and R. Aditi Simha. Hydrodynamics of soft active matter. Rev. Mod. Phys., 85:1143-1189, Jul 2013.

[43] T W Marshall and E J Watson. The analytic solutions of some boundary layer problems in the theory of brownian motion. J. Phys. A, 20(6):1345-1354, apr 1987.

[44] Vijay Narayan, Narayanan Menon, and Sriram Ramaswamy. Nonequilibrium steady states in a vibrated-rod monolayer: tetratic, nematic, and smectic correlations. J. Stat. Mech., 2006(01):P01005.

[45] Vijay Narayan, Sriram Ramaswamy, and Narayanan Menon. Long-lived giant number fluctuations in a swarming granular nematic. Science, 317(5834):105, 2007.

[46] Daniel Needleman and Zvonimir Dogic. Active matter at the interface between materials science and cell biology. Nat. Rev. Mater., 2(9):17048, 2017.

[47] Ran Ni, Martien A. Cohen Stuart, and Peter G. Bolhuis. Tunable long range forces mediated by self-propelled colloidal hard spheres. Phys. Rev. Lett., 114:018302, Jan 2015.

[48] Ran Ni, Martien A. Cohen Stuart, Marjolein Dijkstra, and Peter G. Bolhuis. Crystallizing 
hard-sphere glasses by doping with active particles. Soft Matter, 10(35):6609-6613, 2014.

[49] Jeremie Palacci, Stefano Sacanna, Asher Preska Steinberg, David J. Pine, and Paul M. Chaikin. Living Crystals of Light-Activated Colloidal Surfers. Science, 339(6122):936, 2013.

[50] Sriram Ramaswamy. The mechanics and statistics of active matter. Annu. Rev. Condens. Matter Phys., 1(1):323-345, 2010.

[51] Sriram Ramaswamy. Active matter. J. Stat. Mech., 2017(5):054002, May 2017.

[52] D. Ray, C. Reichhardt, and C. J. Olson Reichhardt. Casimir effect in active matter systems. Phys. Rev. E, 90(1), July 2014.

[53] Gabriel S. Redner, Michael F. Hagan, and Aparna Baskaran. Structure and Dynamics of a Phase-Separating Active Colloidal Fluid. Phys. Rev. Lett., 110:055701, 2013.

[54] Gabriel S. Redner, Caleb G. Wagner, Aparna Baskaran, and Michael F. Hagan. Classical nucleation theory description of active colloid assembly. Phys. Rev. Lett., 117:148002, Sep 2016.

[55] C.J. Olson Reichhardt and C. Reichhardt. Ratchet effects in active matter systems. Annu. Rev. Condens. Matter Phys., 8(1):51-75, March 2017.

[56] David Saintillan. Rheology of active fluids. Annu. Rev. Fluid Mech., 50(1):563-592, 2018.

[57] *Tim Sanchez, Daniel T. N. Chen, Stephen J. DeCamp, Michael Heymann, and Zvonimir Dogic. Spontaneous motion in hierarchically assembled active matter. Nature, 491(7424):431, 2012.

[58] William Savoie, Sarah Cannon, Joshua J. Daymude, Ross Warkentin, Shengkai Li, Andréa W. Richa, Dana Randall, and Daniel I. Goldman. Phototactic supersmarticles. Artificial Life and Robotics, 23(4):459-468, September 2018.

[59] Christian Scholz, Soudeh Jahanshahi, Anton Ldov, and Hartmut Löwen. Inertial delay of self-propelled particles. Nat. Commun., 9(1):5156, 2018. 
[60] Udo Seifert. From stochastic thermodynamics to thermodynamic inference. Annu. Rev. Condens. Matter Phys., 10(1):171-192, 2019.

[61] Francisco J. Sevilla and Mario Sandoval. Smoluchowski diffusion equation for active brownian swimmers. Phys. Rev. E, 91:052150, May 2015.

[62] Granville Sewell. Solving PDEs in non-rectangular 3d regions using a collocation finite element method. Adv. Eng. Software, 41(5):748-753, May 2010.

[63] Granville Sewell. Solving Partial Differential Equation Applications with PDE2D. Wiley, 2018.

[64] A. P. Solon, Y. Fily, A. Baskaran, M. E. Cates, Y. Kafri, M. Kardar, and J. Tailleur. Pressure is not a state function for generic active fluids. Nat. Phys., 11:673-678, 2015.

[65] Joakim Stenhammar, Davide Marenduzzo, Rosalind J Allen, and Michael E Cates. Phase behaviour of active Brownian particles: the role of dimensionality. Soft Matter, 10(10):1489, 2014.

[66] Joakim Stenhammar, Adriano Tiribocchi, Rosalind J. Allen, Davide Marenduzzo, and Michael E. Cates. Continuum theory of phase separation kinetics for active brownian particles. Phys. Rev. Lett., 111:145702, 2013.

[67] Joakim Stenhammar, Raphael Wittkowski, Davide Marenduzzo, and Michael E. Cates. Activity-Induced Phase Separation and Self-Assembly in Mixtures of Active and Passive Particles. Phys. Rev. Lett., 114:018301, 2015.

[68] Henning Struchtrup and Manuel Torrilhon. Regularization of grad's 13 moment equations: Derivation and linear analysis. Phys. Fluids, 15(9):2668-2680, August 2003.

[69] Michael Tennenbaum, Zhongyang Liu, David Hu, and Alberto Fernandez-Nieves. Mechanics of fire ant aggregations. Nat. Mater., 15(1):54-59, October 2015.

[70] Kasper van der Vaart, Michael Sinhuber, Andrew M. Reynolds, and Nicholas T. Ouellette. Mechanical spectroscopy of insect swarms. Sci. Adv., 5(7):eaaw9305, July 2019. 
[71] Andrea Villa-Torrealba, Cristóbal Chávez-Raby, Pablo de Castro, and Rodrigo Soto. Runand-tumble bacteria slowly approaching the diffusive regime. Phys. Rev. E, 101(6), June 2020.

[72] Giovanni Volpe, Ivo Buttinoni, Dominik Vogt, Hans-Jürgen Kümmerer, and Clemens Bechinger. Microswimmers in patterned environments. Soft Matter, 7(19):8810, 2011.

[73] Caleb G Wagner and Richard Beals. Constructing solutions to two-way diffusion problems. J. Phys. A, 52(11):115204, feb 2019.

[74] Caleb G Wagner, Michael F Hagan, and Aparna Baskaran. Steady-state distributions of ideal active brownian particles under confinement and forcing. J. Stat. Mech., 2017(4):043203, 2017.

[75] Caleb G. Wagner. Mathematics of nonequilibrium steady states in dilute active matter. PhD thesis, 2020.

[76] Lee Walsh and Narayanan Menon. Ordering and dynamics of vibrated hard squares. J. Stat. Mech., 2016(8):083302, August 2016.

[77] Lee Walsh, Caleb G. Wagner, Sarah Schlossberg, Christopher Olson, Aparna Baskaran, and Narayanan Menon. Noise and diffusion of a vibrated self-propelled granular particle. Soft Matter, 13:8964-8968, 2017.

[78] M. B. Wan, C. J. Olson Reichhardt, Z. Nussinov, and C. Reichhardt. Rectification of swimming bacteria and self-driven particle systems by arrays of asymmetric barriers. Phys. Rev. Lett., 101:018102, Jul 2008.

[79] H. H. Wensink and H. Löwen. Aggregation of self-propelled colloidal rods near confining walls. Phys. Rev. E, 78:031409, Sep 2008.

[80] M. E. Widder and U. M. Titulaer. The kinetic boundary layer around an absorbing sphere and the growth of small droplets. J. Stat. Phys., 55(5):1109-1128, Jun 1989.

[81] Adam Wysocki, Roland G. Winkler, and Gerhard Gompper. Cooperative motion of active 
brownian spheres in three-dimensional dense suspensions. EPL (Europhysics Letters), 105(4):48004, February 2014.

[82] Mahdi Zaeifi Yamchi and Ali Naji. Effective interactions between inclusions in an active bath. J. Chem. Phys., 147(19):194901, November 2017.

[83] Natsuhiko Yoshinaga. Simple models of self-propelled colloids and liquid drops: From individual motion to collective behaviors. Journal of the Physical Society of Japan, 86(10):101009, October 2017.

[84] Andreas Zöttl and Holger Stark. Emergent behavior in active colloids. J. Phys. Condens. Matter, 28(25):253001, May 2016.

[85] P.F. Zweifel. Neutron transport theory. In E. Inonu and P.F. Zweifel, editors, Developments in Transport Theory, pages 73-111. Academic Press, 1967. 